\title{
Role of Alström syndrome 1 in the regulation of blood pressure and renal function
}

\author{
Ankita Bachhawat Jaykumar,, ${ }^{1,2}$ Paulo S. Caceres, ${ }^{1,2}$ Keyona N. King-Medina,, ${ }^{1,2}$ Tang-Dong Liao, ${ }^{1}$ \\ Indrani Datta, ${ }^{3,4}$ Dipak Maskey, ${ }^{1}$ Jürgen K. Naggert, ${ }^{5}$ Mariela Mendez, ${ }^{1}$ William H. Beierwaltes, ${ }^{1,2}$ \\ and Pablo A. Ortiz', \\ ${ }^{1} H y p e r t e n s i o n$ and Vascular Research Division, Department of Internal Medicine, Henry Ford Hospital, Detroit, Michigan, \\ USA. ${ }^{2}$ Department of Physiology, Wayne State University School of Medicine, Detroit, Michigan, USA. ${ }^{3}$ Department of Public \\ Health Sciences and ${ }^{4}$ Center for Bioinformatics, Henry Ford Health System, Detroit, Michigan, USA. ${ }^{5}$ The Jackson Laboratory, \\ Bar Harbor, Maine, USA.
}

Elevated blood pressure (BP) and renal dysfunction are complex traits representing major global health problems. Single nucleotide polymorphisms identified by genome-wide association studies have identified the Alström syndrome 1 (ALMS1) gene locus to render susceptibility for renal dysfunction, hypertension, and chronic kidney disease (CKD). Mutations in the ALMS1 gene in humans causes Alström syndrome, characterized by progressive metabolic alterations including hypertension and CKD. Despite compelling genetic evidence, the underlying biological mechanism by which mutations in the ALMS1 gene lead to the above-mentioned pathophysiology is not understood. We modeled this effect in a KO rat model and showed that ALMS1 genetic deletion leads to hypertension. We demonstrate that the link between ALMS1 and hypertension involves the activation of the renal $\mathrm{Na}^{+} / \mathrm{K}^{+} / 2 \mathrm{Cl}^{-}$cotransporter $\mathrm{NKCC2}$, mediated by regulation of its endocytosis. Our findings establish a link between the genetic susceptibility to hypertension, CKD, and the expression of ALMS1 through its role in a salt-reabsorbing tubular segment of the kidney. These data point to ALMS1 as a potentially novel gene involved in BP and renal function regulation.

Conflict of interest: The authors have declared that no conflict of interest exists.

License: Copyright 2018, American Society for Clinical Investigation.

Submitted: June 28, 2017 Accepted: September 26, 2018 Published: November 2, 2018

\section{Reference information:} JCI Insight. 2018;3(21):e95076. https://doi.org/10.1172/jici. insight.95076.

\section{Introduction}

Hypertension is a complex polygenic disease in which multiple genes contribute to alter multiple organ function to elevate blood pressure. Our current understanding of the complex genetic architecture of hypertension relies heavily on the identification of mutations causing rare inherited disorders and the discovery of several susceptibility loci through population-based association studies. Such studies have allowed us to identify genetic regions associated with hypertension and its causes and complications like renal dysfunction, chronic kidney disease (CKD), or decreased vascular reactivity, among others.

A recent genome-wide association study (GWAS) in 67,000 White individuals has identified susceptibility variants associated with decreased renal function and CKD in the Alström syndrome 1 (ALMS1) gene locus (1). This gene locus was also identified for genetic risk associated with decreased estimated glomerular filtration rate (eGFR) and CKD by an independent consortium (2). The association between decreased eGFR, CKD, and variant rs13538 in the ALMS1 gene was found with a high minor allele frequency of $22 \%$ in the studied population $(1,3)$. Single nucleotide polymorphisms (SNPs) in the $A L M S 1$ gene were also associated with hypertension, increased pulse pressure, and pulse rate in a multipoint linkage analysis in primary sibling samples of African American, White, and Mexican population (4). In humans, loss-offunction mutations in the ALMS1 gene causes Alström syndrome characterized by cardiomyopathy, hypertension, obesity, and type 2 diabetes. However, little is known about the function of the ALMS1 protein. In some cells, ALMS1 protein is present in the basal body of the cilia. However, cultured fibroblasts from Alström syndrome patients had normal cilia but had defective trafficking of transferrin (5). Therefore, the pathology observed in these patients may be due to defects in intracellular trafficking (5-7). ALMS1 was recently shown to be involved in intracellular trafficking of glucose transporter type 4 (GLUT4) (8). The carboxyl-terminus of ALMS1 (C-ALMS1) interacts with proteins such as $\alpha$-actinin $1 / 4$, Myosin $\mathrm{Vb}$, and 
Rad50 interactor 1 (RINT1), known to be involved in endocytosis (5, 9-12). A gene-trapped ALMS1 mouse model recapitulated age-dependent metabolic syndrome, similar to that observed in Alström syndrome patients (6). However, the role of ALMS1 in BP regulation, renal function, or sodium homeostasis has not been studied, to our knowledge, despite genetic studies supporting a role in $\mathrm{CKD}$.

We found that ALMS1 interacted with the renal $\mathrm{Na}^{+} / \mathrm{K}^{+} / 2 \mathrm{Cl}^{-}$cotransporter (NKCC2), an apical $\mathrm{NaCl}$ transporter expressed in the thick ascending limb of the loop of Henle (thick ascending limb; TAL). Enhanced NKCC2 activity is associated with hypertension in humans $(13,14)$ and animal models $(15)$, and NKCC2-mediated $\mathrm{NaCl}$ reabsorption is known to be determined by its trafficking to the apical membrane $(15,16)$. Here, we describe a potentially novel interaction of ALMS1 with a region of the carboxyl-terminus of NKCC2 (C2-NKCC2). Protein-to-protein interactions at C2-NKCC2 have been described to regulate NKCC2 endocytosis $(17,18)$, and we show that endocytosis contributes to maintaining steady-state levels of $\mathrm{NKCC} 2$ at the apical membrane and $\mathrm{NaCl}$ reabsorption by the TAL (19). Since ALMS1 interacts with C2-NKCC2, we hypothesized that ALMS1 mediates NKCC2 endocytosis and contributes to $\mathrm{NaCl}$ reabsorption by the TAL and, therefore, is involved in the control of BP.

\section{Results}

ALMS1 interacts with a domain in NKCC2 important for apical trafficking. Only 4 proteins are known to bind the $\sim 400$ amino acid-long, intracellular carboxyl-terminus of NKCC2. Within the intracellular carboxyl terminus, there is a unique 71-amino acid stretch (C2-NKCC2) shown to be important for NKCC2 apical trafficking (18). To identify proteins expressed in the TAL that interact with this domain, we followed an in vitro-targeted proteomics approach. We designed a glutathione-s-transferase-fusion (GST-fusion) construct with the 71 amino acids of the rat C2-NKCC2 (GST-C2-NKCC2) and used the purified protein as a bait to pull down proteins from lysates obtained from isolated rat medullary TALs. We characterized the interacting proteins by liquid chromatography (LC), followed by mass spectrometry (MS). Data analysis for proteins of unknown function in the kidney revealed the presence of unique peptides that correspond to ALMS1 (Figure 1A) and were not present in control GST pull down, indicating that C2-NKCC2 interacts with ALMS1. To study the localization of ALMS1 in the kidney, we generated a new antibody against the carboxyl terminus of ALMS1 (Supplemental Figure 1; supplemental material available online with this article; https://doi.org/10.1172/jci.insight.95076DS1) and performed immunofluorescent labeling of ALMS1 in rat kidney transverse sections (Figure 1B). We observed that ALMS1 is expressed in TALs indicated by coimmunolabeling for NKCC2.

Thus, we hypothesized that ALMS1 interacts with NKCC2 to regulate its apical surface trafficking, its activity, and - therefore - BP. To test this, we expressed full-length eGFP-NKCCC2 in normal rat kidney (NRK52E) cells and found that GFP immunoprecipitates with endogenously expressed ALMS1 (Figure 1C). In a yeast two-hybrid screen, the C-ALMS1 was shown to interact with proteins such as Myo$\sin \mathrm{Vb}$, and actin binding protein $\alpha$-actinin, thereby linking ALMS1 to protein trafficking (5). We studied if C-ALMS1 also interacts with NKCC2. To test this, we used truncated portions of ALMS1 C-terminus (C-ALMS1 A, C-ALMS1 B) fused to GST (GST-C-ALMS1 A/B) as baits in the GST pull-down assay in lysates from isolated TALs. We identified a region in ALMS1 that is able to pull down full-length NKCC2 (Figure 1D). We also identified several other proteins by MS analysis that were pulled down by C-ALMS1 A/B. Ingenuity pathway analysis suggested that many of these interacting partners of ALMS1 are involved in endocytosis $(12,20-31)$ (Supplemental Table 1) and may, together, form a network for the NKCC2 endocytic machinery (Figure 1E).

ALMS1 colocalizes with NKCC2 in the TAL. Expression and function of NKCC2 in the TAL has been widely studied. Here, we show that ALMS1 is also expressed in isolated rat TALs via Western blot (Figure 2A). To study the localization pattern of ALMS1 in the TAL, we performed immunolabeling for ALMS1 and confocal microscopy in isolated perfused rat TALs (Figure 2B). Similar to the expression pattern of NKCC2 in rat TAL (32), ALMS1 was found at the apical membrane and subapical space. To study whether ALMS1 is involved in NKCC2 endocytosis, we measured whether it colocalizes with internalized NKCC2 in primary cultures of rat TAL cells. For this, we labeled surface NKCC 2 at $4^{\circ} \mathrm{C}$ with an antibody that recognizes an epitope in an extracellular loop (33). Following a 30-minute period of endocytosis at $37^{\circ} \mathrm{C}$, we fixed the cells and labeled ALMS1. We observed that a small fraction of ALMS1 colocalized with NKCC2 in intracellular vesicles (Figure 2C). The colocalizing vesicles where NKCC2 and ALMS1 colocalize were identified by measuring colocalizing pixels with a Mander's overlap coefficient $\geq 0.95$. In fixed kidney sections, ALMS1 is located in the apical and 
A

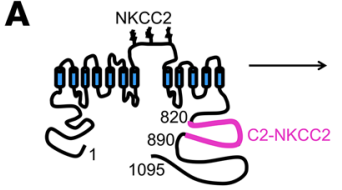

GST (GST only: control) + TAL

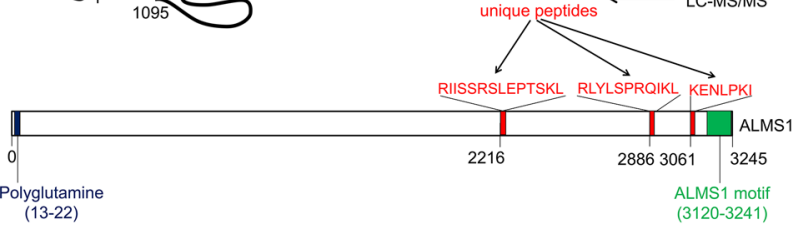

D
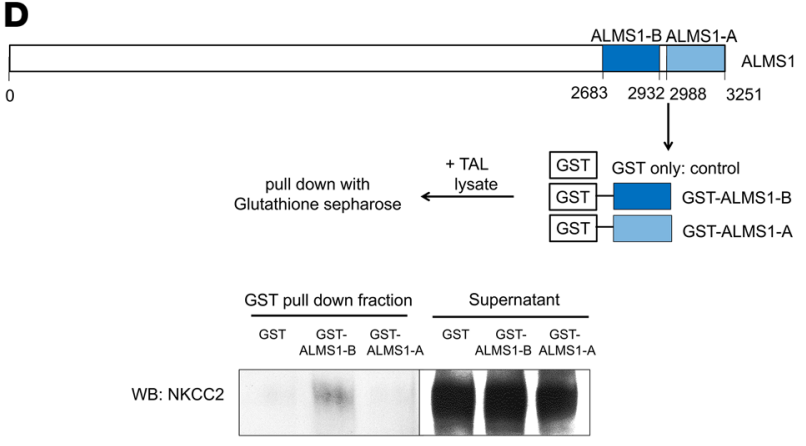

B
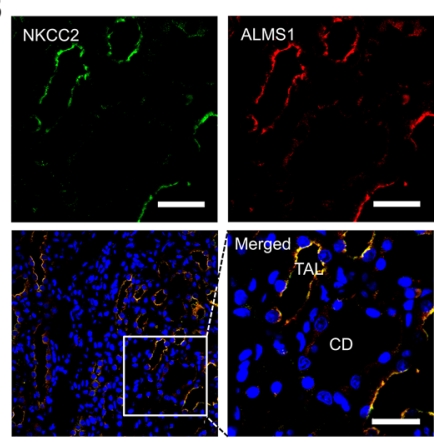

E

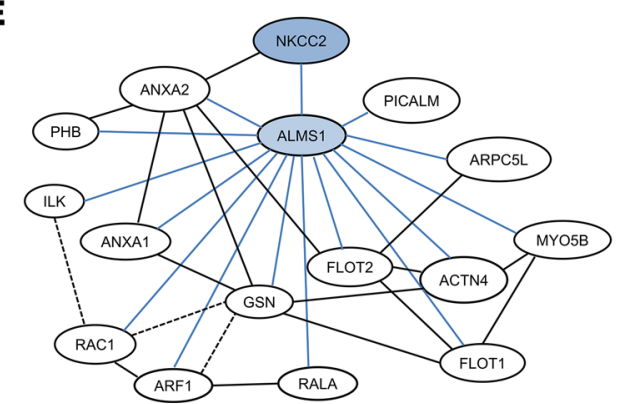

Figure 1. Interaction of ALMS1 with C2-NKCC2. (A) Representation of C2-NKCC2 domain (pink) and unique peptides corresponding to ALMS1 (red) picked up by liquid chromatography-mass spectrometry in glutathione-s-transferase (CST) pull-down assay using CST-carboxyl-terminus of NKCC2 fusion protein (CST-C2-NKCC2) or GST alone (control) as baits in rat thick ascending limb (TAL) lysates. (B) Immunofluorescent labeling for NKCC2 (green) and ALMS1 (red) in paraffin embedded rat kidney sections, indicating expression and colocalization of NKCC2 and ALMS1 in rat TAL. CD, collecting duct; $n=3$. Scale bars: $20 \mu \mathrm{m}$. (C) Representative Western blot for immunoprecipitation of ALMS1 with NKCC2 in NRK 52E cells transduced with EGFP or EGFP-NKKC2 adenovirus construct; $n=3$. (D) Western blot representing GST pull down of full-length NKCC2 with GST-truncated carboxyl-terminus ALMS1 fusion protein $\mathrm{B}$ (GST-C-ALMS1 B) as bait in rat TAL lysates; $n=3$. The lanes were run on the same gel but were noncontiguous and contrast changed on the supernatant fraction for visualization of discrete protein bands. (E) Recreated IPA of protein-to-protein interactions identified in GST pull-down assay with GST-carboxyl-terminus ALMS1 fusion proteins A/B (CST-C-ALMS1 A/B) in rat TAL lysates detected by liquid chromatography-mass spectrometry; $n=1$. Solid and broken lines in black indicate direct and indirect protein interactions from IPA, respectively. Solid blue lines indicate protein interactions from mass spectrometry analysis of C-ALMS1 A/B pull-down protein fraction. RAC1, ras-related C3 botulinum toxin substrate 1; FLOT1, flotillin 1; ANXA2, annexin A2; PICALM, phosphatidyl inositol binding clathrin assembly protein; ARPC5L, actin related protein $2 / 3$ complex subunit 5-like; RALA, ras like proto-oncogene A; ARF1, ADP ribosylation factor 1; ILK, integrin linked kinase; PHB, prohibitin; ACTN4, $\alpha$-actinin 4; MYO5B, myosin 5b; GSN, gelsolin. Respective roles of these proteins in regulating endocytosis are defined in Supplemental Table 1.

subapical space of TAL cells, where it partially colocalizes with NKCC2 (Figure 2D; Mander's overlap coefficient $\geq 0.9$ and Pearson's coefficient $\geq 0.6$ ). These data indicate that ALMS1 is located at the apical membrane and subapical space of TALs, where it could mediate or be involved in NKCC2 internalization.

Generation of the ALMS1 genetic deletion rat model and its phenotypic characterization. To study the role of ALMS1 in renal function, an ALMS1-KO rat was generated using zinc finger nuclease gene editing technology in collaboration with the Gene Editing Rat Resource Center (GERRC) at the Medical College of Wisconsin (Madison, Wisconsin, USA). Deletion of a 17-bp sequence in exon 1 of the ALMS1 gene results in a premature stop codon causing deletion of $A L M S 1$ in rats (Figure 3A). Specific primer amplification followed by restriction digestion show heterozygous and homozygous deletions (Figure 3B) in these rats. To verify the KO of ALMS1 in the ALMS1-KO rats, we performed immunofluorescence labeling of NKCC2 and ALMS1 in kidney sections from WT and $A L M S 1-\mathrm{KO}$ rats. As expected, ALMS1 was undetectable in ALMS1-KO rats, whereas NKCC2 was clearly detected, indicating a successful deletion of ALMS1 in ALMS1-KO rats (Figure 3C).

Homozygous ALMS1-KO rats were bred from heterozygous carriers. We observed no major differences in the rats we studied before the age of 12 weeks, except for body weight, which was slightly higher than WT only after 10-11 weeks of age. Blood glucose and plasma insulin were not different between the groups by 12 weeks of age. Gross kidney anatomy was normal in histological sections (Supplemental Figure 2), and ALMS1-KO rats had similar kidney weight, GFR and renal blood flow, plasma osmolality, plasma sodium, potassium, and chloride concentration compared with WT littermate rats at 6-12 weeks of age on a normal salt diet (Table 1). These data indicate that, by this age, ALMS1-KO rats do not significantly develop major metabolic abnormalities or kidney damage. Thus, we used rats of age 6-12 weeks for our studies. 
A

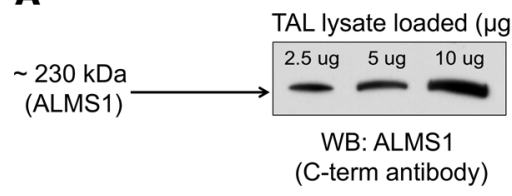

B

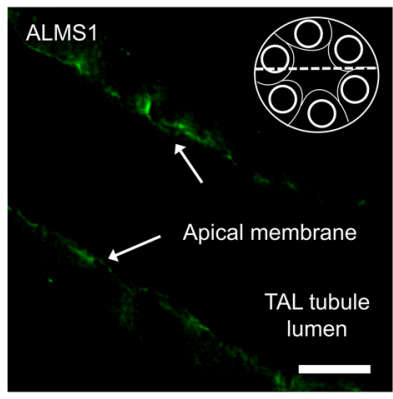

C

Maximum intensity projection

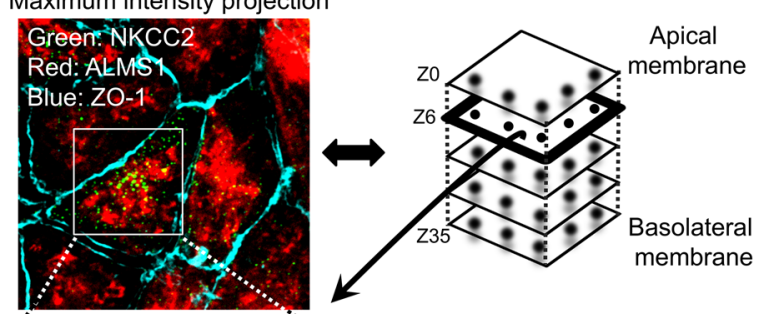

D
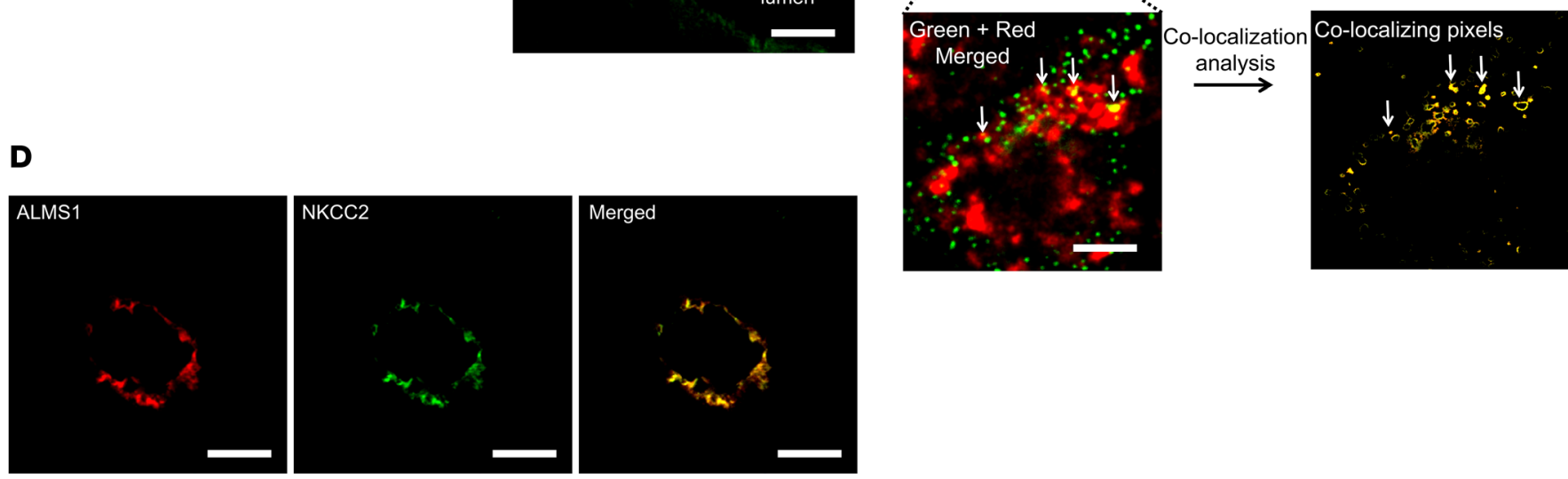

Figure 2. Expression and colocalization of ALMS1 and NKCC2 in rat TAL. (A) Representative immunoblot of ALMS1 indicating ALMS1 protein expression in rat thick ascending limb (TAL) corresponding to $230 \mathrm{kDa}$ protein; $n=4$. (B) Representative image for immunofluorescent labeling of ALMS1 in isolated, perfused TAL, indicating apical and subapical vesicular localization of ALMS1 by confocal imaging in a cross-section of TAL; $n=3$. Scale bars: $5 \mu$ m. (C) Representative image for immunofluorescent labeling of NKCC2 and ALMS1 in rat TAL primary cultured cells; $n=3$. NKCC2 (green); ALMS1 (red); tight junction protein zonula occludens 1 (blue). Merged image from a subapical plane (1.5 $\mu \mathrm{m}$ from the apical plane) showing overlap of internalized NKCC2 and ALMS1 indicated with an arrow. Scale bars: $5 \mu \mathrm{m}$. (D) Representative image for immunofluorescent labeling of NKCC2 (green) and ALMS1 (red) in a single TAL cross-section obtained from perfusion-fixed rat kidney slices $(n=4)$. Scale bars: $10 \mu \mathrm{m}$.

ALMS1-KO rats are hypertensive and salt sensitive. To test our hypothesis that genetic deletion of ALMS1 in rats increases $\mathrm{BP}$, we used 8- to 10-week-old ALMS1-KO rats and WT littermates fed a normal salt diet to measure conscious BP. Radiotelemetric devices were implanted, and 1 week later, BP was monitored for 3 consecutive days. We observed that, during their awake/dark period, systolic BP was higher by $10.5 \mathrm{mmHg}$ in ALMS1-KO rats, and rats were hypertensive (Figure 4A). Systolic BP during the rest/light period was higher by $14 \mathrm{mmHg}$ in $A L M S 1$-KO rats (Figure 4B). The mean BPs of ALMS1-KO rats during their awake and rest periods were also significantly higher by $6 \mathrm{mmHg}$ and $9 \mathrm{mmHg}$, respectively (Figure 4, C and D). As expected, heart rate in ALMS1-KO rats was significantly lower by $25 \mathrm{bpm}$ compared with WT rats (Figure 4E), suggesting compensatory bradycardia to offset hypertension in the ALMS1-KO rats. Additionally, the hypertension observed is not likely related to elevated renin, since plasma renin activity (PRA) was not different between the strains (Table 1). A representative 24-hour telemetry tracing (Figure $4 \mathrm{~F}$ ) for a WT and a $A L M S 1$-KO rat is included. By tail cuff plethysmography, we found that the systolic $\mathrm{BP}$ was higher in $A L M S 1-\mathrm{KO}$ rats on normal salt intake and was increased by $14 \mathrm{mmHg}$ after 7 days of high salt intake, whereas BP did not increase in WT rats on high salt intake. Thus, $A L M S 1-\mathrm{KO}$ rats are hypertensive and more sensitive to high salt intake than WT rats (Figure 4G).

ALMS1-KO rats have a higher NKCC2-mediated NaCl absorption in the TAL. In order to test whether the increase in $\mathrm{BP}$ in $A L M S 1$-KO rats is associated with higher $\mathrm{NaCl}$ reabsorption by the TAL, we measured NKCC2-mediated $\mathrm{NaCl}$ transport in vivo. Firstly, we housed rats in metabolic cages and collected 24-hour urine. At baseline, urine volume was not significantly different between the groups (Figure 5A). However, urine osmolality was 50\% higher in ALMS1-KO rats (Figure 5B). NKCC2 is required for urine concentration such that decreased NKCC2 activity causes dilute urine (34), whereas increased activity increases the interstitial osmolality, enhancing urine concentrating capacity. To measure NKCC2-mediated $\mathrm{NaCl}$ absorption in vivo, we measured the acute diuretic and natriuretic effect of the NKCC2 inhibitor bumetanide. We found that urine volume and urinary sodium excretion after a submaximal dose of bumetanide was $95 \%$ and $120 \%$ higher in ALMS1-KO rats compared with WT rats, respectively (Figure 5, C and D). This indicated that $A L M S 1$-KO rats have higher NKCC2-mediated $\mathrm{NaCl}$ transport. 
A
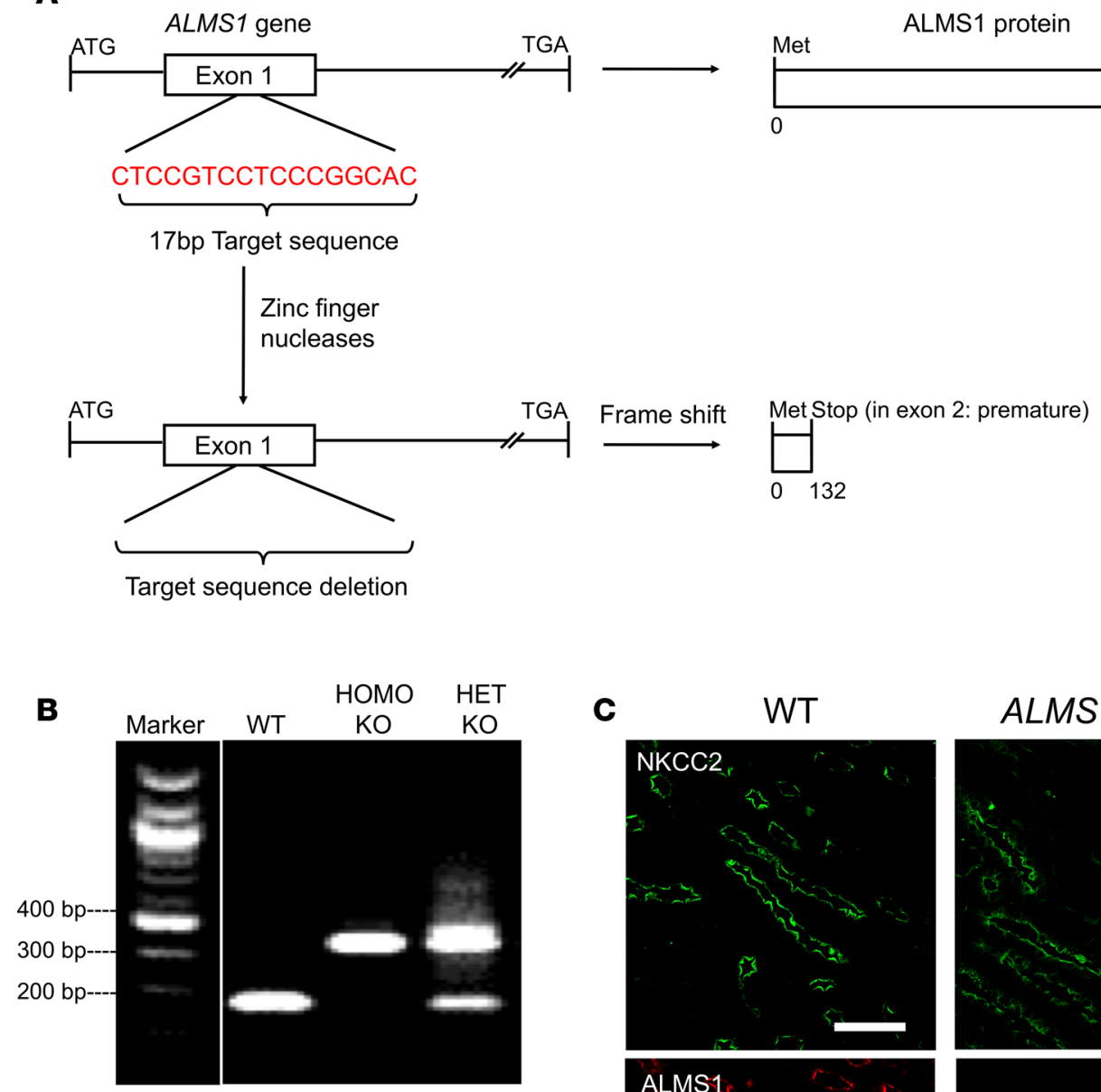

C
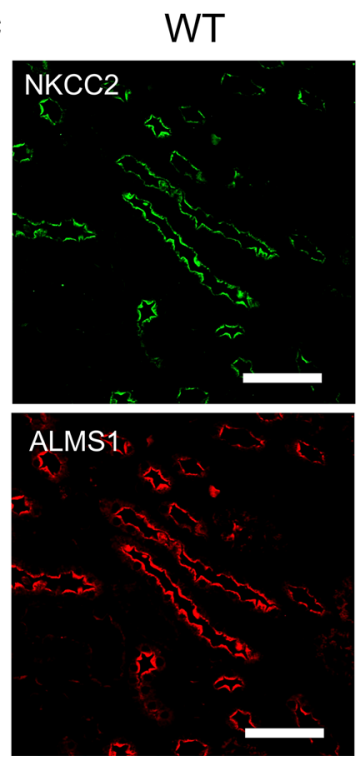

ALMS1 KO
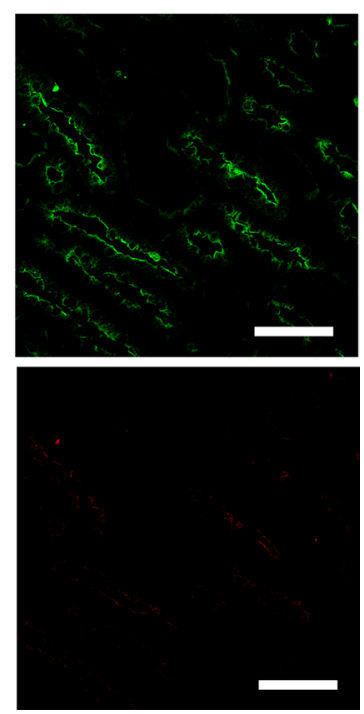

Figure 3. Generation of ALMS1-KO rats and their genotyping. (A) Scheme showing deletion of 17-bp sequence from exon 1 of ALMS1 gene by zinc finger nucleases leading to premature stop codon in exon 2, resulting in KO of ALMS1 in rats. (B) PCR using exon 1-specific primers, followed by Nci1 restriction enzyme digestion, generated 365 bp fragment in homozygous KO and 181 bp and 201 bp fragments in WT and all the above fragments in heterozygous KO. The lanes were run on the same gel but were noncontiguous. (C) Representative image for immunofluorescent labeling of ALMS1 (red) and NKCC2 (green) in paraffin embedded rat kidney sections, indicating KO of ALMS1 in ALMS1-KO rat in rat TAL; $n=3$. Scale bars: $30 \mu \mathrm{m}$.

Higher NKCC2-mediated $\mathrm{NaCl}$ transport may be related to increased $\mathrm{BP}$ by preventing proper $\mathrm{Na}$ excretion. We then tested whether the ability to excrete an acute saline/volume load is decreased in ALMS1-KO rats. For this, we instrumented anesthetized rats and performed a $3 \%$ volume expansion (with respect to body weight) with isotonic saline, and we collected urine at intervals of 30 minutes. We found that the cumulative urine volume and urinary sodium excretion (normalized to the baseline values) after volume expansion was significantly lower in $A L M S 1$-KO rats by $60 \%$ and $75 \%$, respectively (Figure 6, A and B). Taken together, these data indicate that $A L M S 1-\mathrm{KO}$ rats have a higher NKCC2-mediated $\mathrm{NaCl}$ reabsorption and decreased ability to excrete a salt and volume load. These effects may be related to the hypertension observed in these rats.

ALMS1-KO rats have enhanced NKCC2 levels at the apical surface in TAL. The mechanism that causes enhanced NKCC2-mediated $\mathrm{NaCl}$ absorption in $A L M S 1-\mathrm{KO}$ rats is unknown. To test whether genetic deletion of $A L M S 1$ in rats leads to higher NKCC2-mediated $\mathrm{NaCl}$ absorption due to enhanced levels of NKCC2 in the apical surface of TALs, we performed surface biotinylation in TAL suspensions obtained 
Table 1. Measurement of physiological parameters in 6-12 week old WT and ALMS1-KO rats

\begin{tabular}{lcc}
\hline Physiological parameters & WT (6-12 weeks) & ALMS1-Ko (6-12 weeks) \\
Body weight (g) & $309 \pm 10$ & $331 \pm 11^{\mathrm{A}}$ \\
Blood glucose (mg/dl) & $134 \pm 10$ & $138 \pm 12$ \\
Plasma insulin (ng/ml) & $0.45 \pm 0.15$ & $0.7 \pm 0.1$ \\
Kidney weight (g) & $2.75 \pm 0.1$ & $2.53 \pm 0.13$ \\
GFR (ml/min/gkw) & $0.94 \pm 0.16$ & $1.07 \pm 0.36$ \\
Plasma osmolality (mOsm/Kg water) & $322.7 \pm 3.17$ & $327.2 \pm 2.43$ \\
Plasma sodium (mM) & $162.8 \pm 0.6$ & $163.2 \pm 0.6$ \\
Plasma potassium (mM) & $4.48 \pm 0.23$ \\
Plasma chloride (mM) & $96 \pm 0.6$ & $94.3 \pm 0.7$ \\
Plasma renin activity (ng Angl/m/hr) & $2.45 \pm 0.3$ & $1.92 \pm 0.26$
\end{tabular}

Blood glucose, plasma insulin, kidney weight, glomerular filtration rate (GFR), and renal blood flow in WT and ALMS1$\mathrm{KO}$ rats measured at $6-12$ weeks was similar. All values represent mean \pm SEM, and statistical analysis was performed with 2-tailed Student's $t$ test. For plasma insulin and blood glucose, $n=3$; body weight, $n=5$; kidney weight and GFR, (WT) $n=12$, (ALMS1-KO) $n=10$; plasma osmolality, sodium, potassium, and chloride concentration, (WT) $n=9,($ ALMS1KO) $n=6$; plasma renin activity, $n=4$. ${ }^{A} P<0.05$ vs. WT.

from WT and ALMS1-KO rats. Western blot for NKCC2 revealed that ALMS1-KO rats have 70\% higher NKCC2 at the surface of TALs compared with WT (Figure 7A), whereas total NKCC2 expression was not different between the groups (Figure 7B).

To assure that the effect of ALMS1 deletion on surface NKCC2 was due to decreased ALMS1 expression in the renal tubule and not secondary to deletion of $A L M S 1$ in other organs, we silenced ALMS1 in the renal medulla by adenovirus-mediated (Ad-mediated) gene silencing. For this, we injected Ad- $A L M S 1$ shRNA into the outer medulla of the left kidney of normal rats while the right kidney served as the control. Seven days after ALMS1 shRNA injection, we observed an 80\% decrease in ALMS1 mRNA (Figure 7C) and a 60\% decrease in ALMS1 protein expression (Figure 7D) in isolated TALs. Surface NKCC2 was enhanced by $70 \%$ in TALs from $A L M S 1$ shRNA-injected kidney compared with control-injected kidney, while there was no change in total NKCC2 expression (Figure 7, E and F). These data confirm that enhanced surface NKCC2 in the TALs of $A L M S 1-\mathrm{KO}$ rats is mostly caused by deletion of $A L M S 1$ in TALs and not secondary to deletion of $A L M S 1$ in other organs.

Higher surface NKCC2 in TALs of ALMS1-KO rats is due to decreased NKCC2 endocytosis. To test our hypothesis that increased surface NKCC2 in $A L M S 1-\mathrm{KO}$ rats is due to decreased NKCC2 endocytosis in the TAL, we performed a modified surface biotinylation protocol to measure NKCC2 internalization. We observed that the fraction of internalized NKCC2 after warming to $37^{\circ} \mathrm{C}$ was significantly lower in TAL from ALMS1-KO rats (Figure 8), while internalized NKCC2 (measured as percentage of total surface NKCC2) was 55\% lower in ALMS1-KO rats compared with WT. These data indicate a decrease in NKCC2 endocytosis in TALs from ALMS1-KO rats. Therefore, according to our current working model (Figure 9), genetic deletion of $A L M S 1$ in rats leads to a higher surface NKCC2 due to a lower rate of NKCC2 endocytosis, and this mechanism may in part be responsible for higher tubular $\mathrm{NaCl}$ reabsorption, volume expansion, and - therefore - hypertension observed in the ALMS1-KO rats.

\section{Discussion}

In this paper, we describe a potentially novel function of ALMS1 as a regulator of renal $\mathrm{NaCl}$ homeostasis and BP. We found that ALMS1 is expressed along the nephron but seems to be enriched in the TAL, where it interacts with the carboxyl terminus of the apical NKCC2. We characterized a new genetic rat model with deletion of $A L M S 1$. In these $A L M S 1-\mathrm{KO}$ rats, we found higher NKCC2-mediated $\mathrm{NaCl}$ reabsorption, due to elevated surface NKCC2 and decreased NKCC2 endocytosis. Additionally, ALMS1-KO rats exhibited a decreased renal capacity to excrete a volume/salt load and hypertension. Therefore, we conclude that the interaction of ALMS1 with NKCC2 is vital to the process of renal $\mathrm{NaCl}$ reabsorption and, therefore, $\mathrm{BP}$ regulation. This is in part mediated by a role of ALMS1 in NKCC2 endocytosis, which maintains normal surface NKCC2 levels and function. Given the unknown function of ALMS1 in the kidney, and the studies linking SNPs in ALMS1 with decreased renal function (GFR), our study is the first to our knowledge to 
A

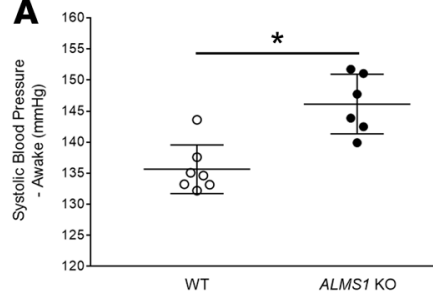

E

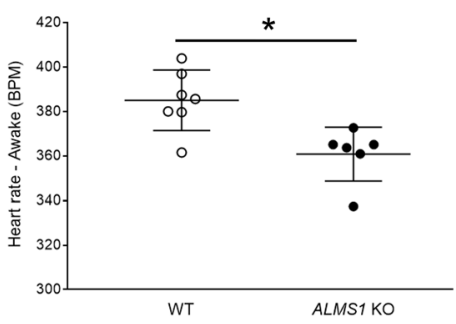

B

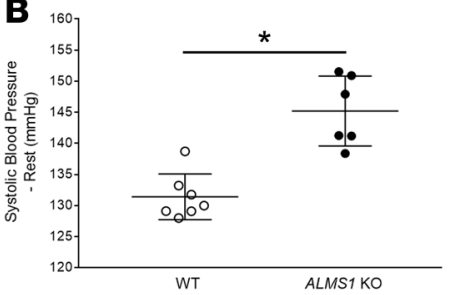

C

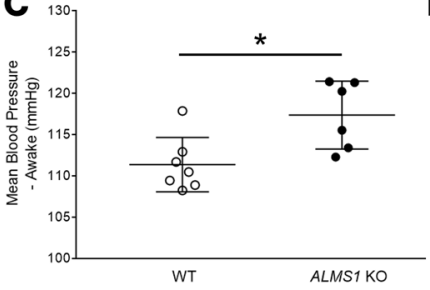

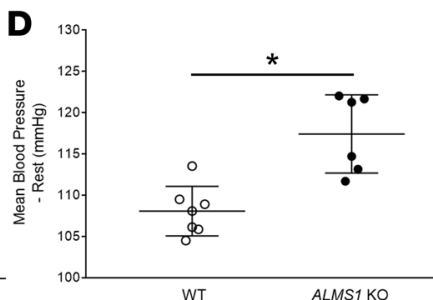

$\mathbf{F}_{2}$
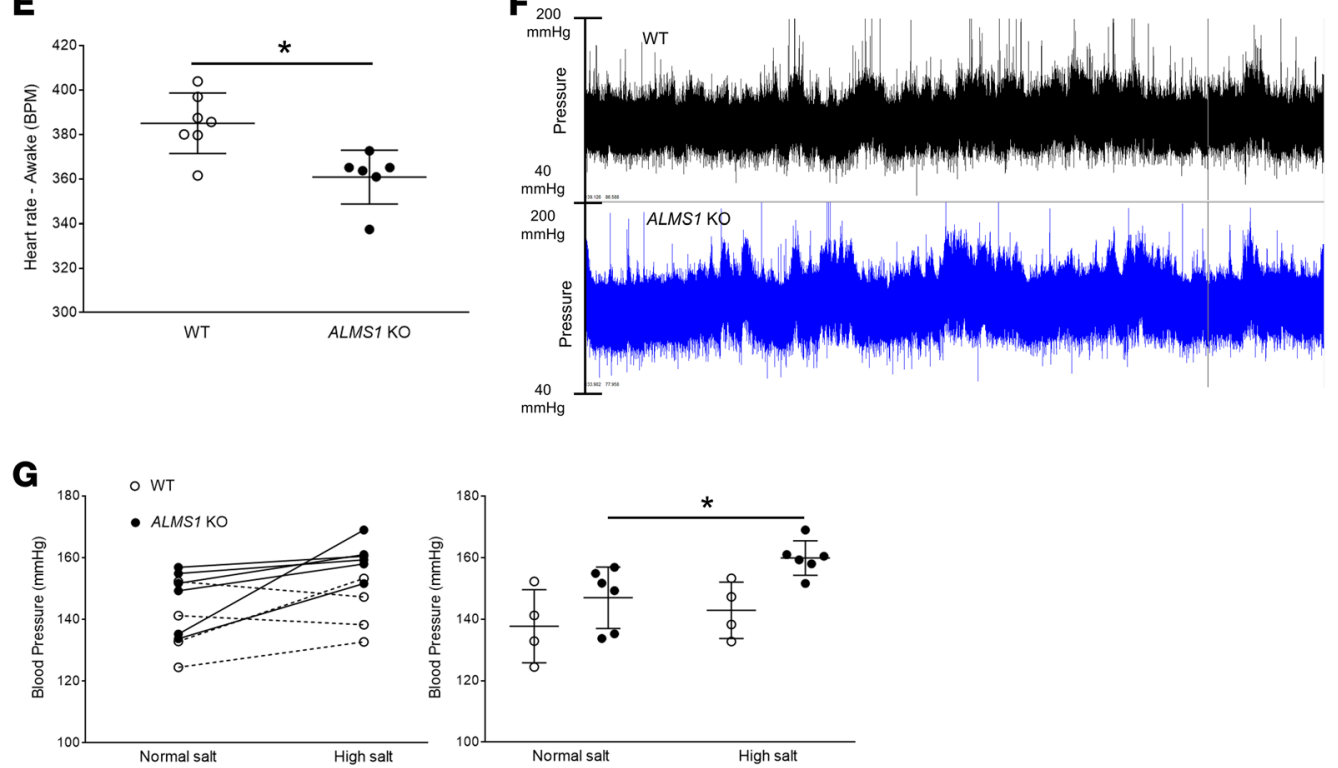

Figure 4. Hypertension in ALMS1-KO rats. (A) On a normal salt diet, systolic blood pressure (SBP) measurement by radiotelemetry in awake rats indicated ALMS1-KO rats are hypertensive (SBP ALMS1-KO, $146.1 \pm 2 \mathrm{mmHg}, n=6$, vs. WT, $135.6 \pm 1.5 \mathrm{mmHg}, n=7$; ${ }^{*} P<0.001$ ). (B) Systolic blood pressure measurement by radiotelemetry indicated that $A L M S 1-K O$ rats are hypertensive during their rest period (SBP ALMS1-KO, 145.2 $\pm 2.3 \mathrm{mmHg}, n=6, \mathrm{vs}$. WT, $131.4 \pm 1.6 \mathrm{mmHg}, n=7$; ${ }^{*} P<0.005$ ). (C) Mean blood pressure (MBP) measured by radiotelemetry in awake rats indicated a higher MBP in ALMS1-KO rats (MBP ALMS1-KO, $117.4 \pm 1.7 \mathrm{mmHg}, n=6$, vs. WT, $\left.111.4 \pm 1.3 \mathrm{mmHg}, n=7 ;{ }^{*} P<0.01\right)$. (D) Mean blood pressure (MBP) measured by radiotelemetry in rats indicated higher MBP in ALMS1-KO during their rest period (MBP ALMS1-KO, $117.4 \pm 2 \mathrm{mmHg}, n=6$, vs. WT, $108.1 \pm 1.3 \mathrm{mmHg}, n=7 ;{ }^{*} P<0.01$ ). (E) Heart rate (HR) measured in awake rats indicated a lower HR in ALMS1-KO rats (HR ALMS1-KO, 360.8 $\pm 5.2 \mathrm{bpm}, n=6, \mathrm{vs}$. WT, $385 \pm 5.6 \mathrm{bpm}, n=7 ;{ }^{*} P<0.01$ ). (F) Representative 24-hour telemetry tracing for 1 WT and ALMS1-KO rat fed with normal salt diet. (G) Systolic blood pressure measurement in awake rats during normal salt (NS) intake and after 7 days on high salt (HS) intake (NS ALMS1-KO, $149.7 \pm 4.76 \mathrm{mmHg}$, vs. HS ALMS1-KO, $162.86 \pm 3.82 \mathrm{mmHg}, n=6$; ${ }^{*} P<0.05$ ) and (NS WT, $137 \pm 5.2 \mathrm{mmHg}, n=6$, vs. HS WT, $142.5 \pm 4 \mathrm{mmHg}, n=4$ ). Both graphs are different representations of the same data set. Values represent mean \pm SEM, and statistical analysis was performed with Student's 2-tailed $t$ test and 2-way ANOVA (salt sensitivity).

suggest that a role of ALMS1 in tubular $\mathrm{NaCl}$ reabsorption and hypertension may contribute to decreasing renal function in patients with decreased ALMS1 function/expression. Interestingly, our data also suggests that hypertension observed in patients with Alström syndrome may be loop-diuretic responsive.

The functional role of ALMS1 in the kidney has not been studied, to our knowledge. However, CKD and hypertension are common in Alström syndrome patients $(35,36)$. SNPs in the ALMS1 gene have been associated with hypertension, increased pulse pressure, and increased pulse rate in a multipoint linkage analysis in primary sibling samples of African American, White, and Mexican population (4). A GWAS (1, 3) found genetic variants in ALMS1 associated with decreased kidney function and hypertension (4). The above-mentioned studies $(1,3)$, along with other studies by independent consortia (37-41), found susceptibility variants in uromodulin (UMOD, Tamm-Horsfall protein), another protein highly expressed in the TAL, to be associated with decreased renal function and hypertension. A functional link between NKCC2 and UMOD was established showing that UMOD may play a role in BP regulation via regulating NKCC2 activity (42). While the molecular function of UMOD has been well studied, it is not known if there is an interaction between UMOD and ALMS1. We did not detect UMOD in our pull-downs with C2-NKCC2 or C-ALMS1, suggesting that they may be linked to renal function through the regulation of NKCC2 activity rather than interacting directly. ALMS1 is encoded by a very large gene, and additional studies will be required to study if SNPs in the region coding for its carboxyl terminus show stronger association 
A
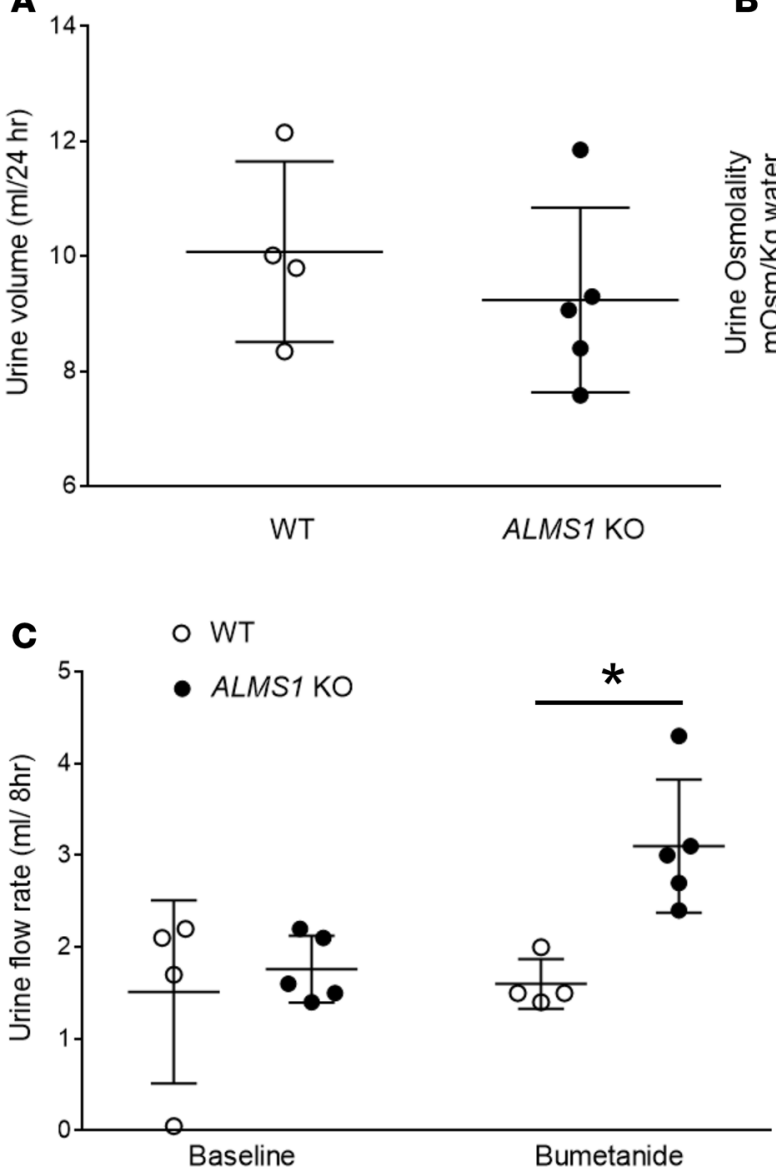

B

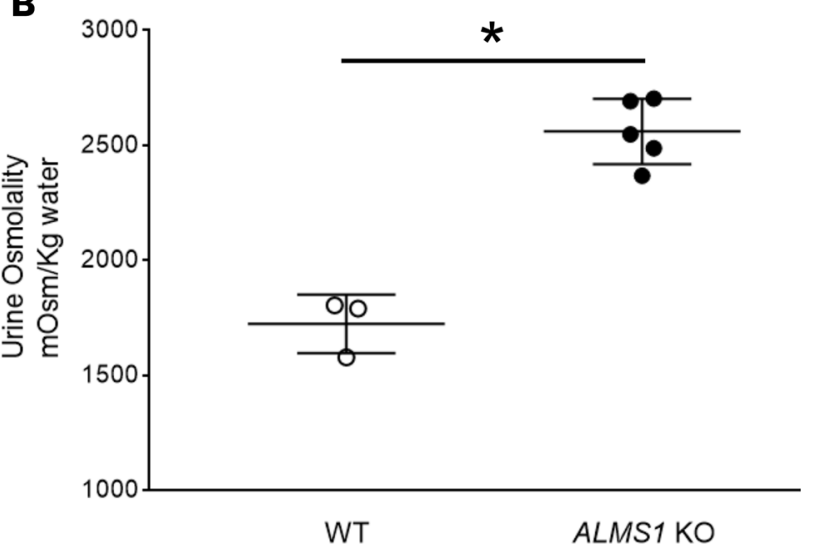

D

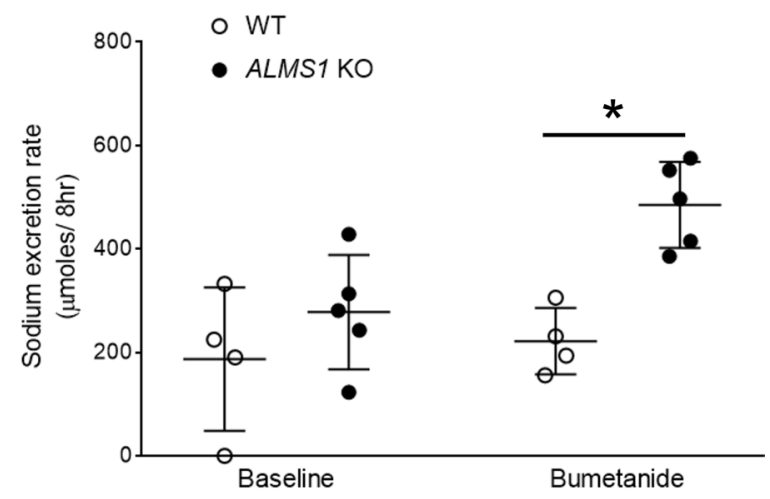

Figure 5. ALMS1-KO rats have higher NKCC2-mediated NaCl absorption in thick ascending limb (TAL). (A) Twenty-four-hour urine volume collected by placing the rats in metabolic cages was not different between the groups (ALMS1-KO, $9.24 \pm 0.72 \mathrm{ml}, n=5$, vs. WT, $10.08 \pm 0.78 \mathrm{ml}, n=4$ ). (B) Urine osmolality measured in 24-hour urine collected sample was higher in ALMS1-KO rats (ALMS1-KO, 2,560 $\pm 63.4 \mathrm{mOsm} / \mathrm{kg}$ water, $n=5$, vs. WT, 1,724 $\pm 73.1 \mathrm{mOsm} /$ $\mathrm{kg}$ water, $n=3$; ${ }^{*} P<0.005$ ). (C) Urine volume measured 8 hours after $5 \mathrm{mg} / \mathrm{kg}$ bumetanide (submaximal dose) treatment was higher in ALMS1-KO rats (ALMS1-KO, $3.1 \pm 0.32 \mathrm{ml} / 8$ hours, $n=5$, vs. WT, $1.6 \pm 0.13 \mathrm{ml} / 8$ hours, $n=4 ;{ }^{*} P<0.025 \mathrm{vs}$. WT). (D) Urinary sodium excretion measured 8 hours after $5 \mathrm{mg} /$ $\mathrm{kg}$ bumetanide treatment was higher in ALMS1-KO rats (ALMS1-KO, $485.2 \pm 37.1 \mu$ moles/8 hours, $n=5$, vs. WT, $221.8 \pm 32 \mu$ moles/8 hours, $n=4$; ${ }^{*} P<0.025$ vs. WT). Values represent mean \pm SEM, and statistical analysis was performed with 2-tailed Student's $t$ test and 2-way ANOVA.

with hypertension or renal dysfunction. Our data in ALMS1-KO rats suggest that any SNP (not just in the region coding for the carboxyl-terminus) that decreases expression or function of ALMS1 could potentially increase renal $\mathrm{NaCl}$ absorption and, therefore, contribute to hypertension in the general population. In addition, the function of ALMS1 in the control of vascular reactivity and sympathetic nervous system activity is unclear, and they may be involved in the hypertension observed in ALMS1-KO rats. Thus, additional studies should focus on this.

Little is known about the function of ALMS1 in other organs. ALMS1 seems to be involved in the development of obesity, since Alström syndrome patients develop early-onset obesity and since ALMS1-targeted mutations in mice leads to the development of age-dependent obesity, insulin resistance, diabetes, and hepatic steatosis between the ages of 18 and 21 weeks (8). In our study, we used young-adult rats between the age of 6 and 12 weeks to avoid the potential effect of other confounding factors on the measurement of $\mathrm{BP}$ and renal function. At this age, body weight was slightly higher in ALMS1-KO rats, but metabolic parameters were normal (Table 1). In addition, ALMS1 may be involved in the development of insulin resistance through its actions on GLUT4. It is unclear whether ALMS1 regulates insulinstimulated GLUT4 endocytosis or recycling (8). However, the carboxyl terminus of ALMS1 interacts with proteins that form the endocytic machinery (e.g., $\alpha$-actinin 4, Myosin Vb, RINT1) (5, 9-12). Thus, ALMS1 may be involved in the development of insulin resistance through the regulation of insulin-stimulated GLUT4 trafficking. At the age we studied our rats (6-12 weeks), they had normal plasma glucose and insulin. However, it is likely that $A L M S 1$-KO rats will develop metabolic syndrome as they age. 

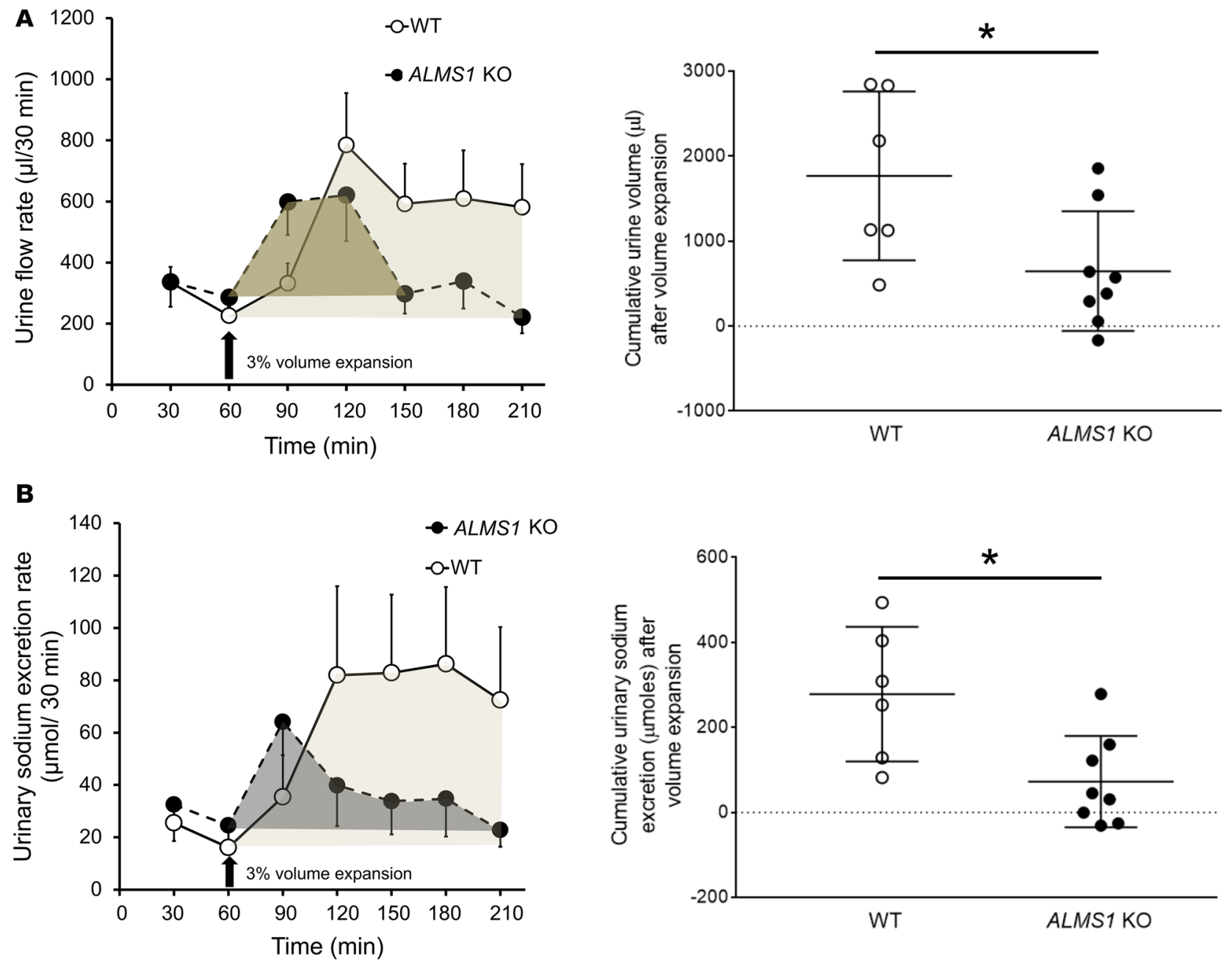

Figure 6. Urinary excretory capacity in response to volume/ salt load with isotonic saline is decreased in ALMS1-KO rats. (A) Cumulative urine volume (adjusted to baseline) upon $3 \%$ volume expansion measured between time points 60 and 210 minutes was decreased in ALMS1-KO rats (ALMS1-KO, 0.7 \pm 0.24 $\mathrm{ml}, n=8$, vs. WT: $1.77 \pm 0.4 \mathrm{ml}, n=6$; ${ }^{*} P<0.05$ ). (B) Cumulative urinary sodium excretion (adjusted to baseline) upon $3 \%$ volume expansion measured between time points 60 and 210 minutes was decreased in ALMS1-KO rats (ALMS1-KO, $72.5 \pm 37.9 \mu$ moles, $n=8$, vs. WT, $278.4 \pm 64.5 \mu$ moles, $n=6$; ${ }^{*} P<0.05$ ). Values represent mean \pm SEM, and post hoc statistical analysis was performed using the Bonferroni correction for multiple comparisons and 2 -tailed Student's $t$ test.

ALMS1 contains an ALMS1 motif in its C-terminus that is reported to be important for primary cilium assembly (43). In cultured cells, ALMS1 has been localized to the ciliary basal body. Li et al. reported that shRNA-mediated knockdown of $A L M S 1$ in a mouse inner medullary collecting duct (mIMCD3) cell line resulted in stunted cilia (44). However, most data do not support a role for ALMS1 in ciliary function. For example, primary cilia of renal collecting duct tubules from ALMS1 mutant mice appeared normal in size and number (6). Similarly, cultured fibroblasts from Alström syndrome patients exhibited normal cilia but showed defects in membrane trafficking (5). Thus, the role of ALMS1 in maintaining ciliary function is unclear and debatable. To study this, we labeled cilia in kidney sections and measured ciliary length in renal tubules. We found that cilia length appeared normal in ALMS1-KO rats and similar to that of WT rats (Supplemental Figure 3; quantification not shown). Thus, our data indicate that the renal phenotype observed in $A L M S 1-\mathrm{KO}$ rats is likely independent from ciliary length and primarily due to defects in NKCC2 endocytosis.

C2-NKCC2 is a 71-amino acid domain in carboxyl-terminus of NKCC2 known to be important for apical trafficking of NKCC2. In this study, ALMS1 was identified as an interacting partner with this domain and involved in some part of the NKCC2 internalization process. A previous study had identified that MAL/VIP17 interacts with this domain in NKCC2 in LLC-PK1 cell lines and decreases NKCC2 internalization by retaining NKCC2 at the surface (17). These data suggest that C2-NKCC2 may be an important regulatory domain involved in modulating NKCC2 surface expression mediated via 
A

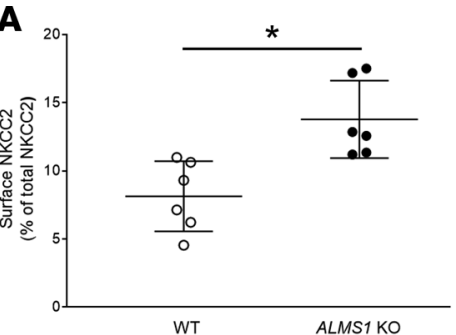

B

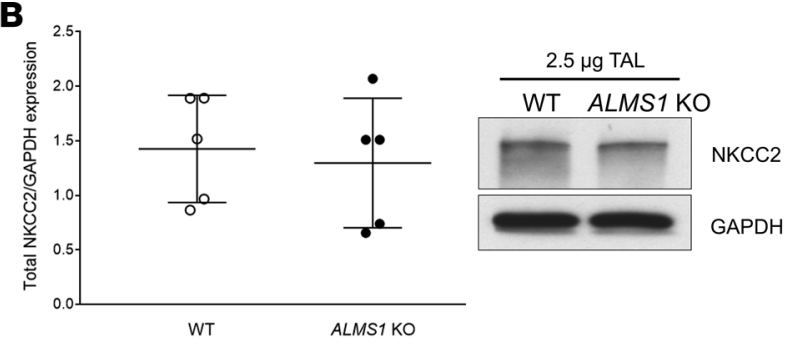

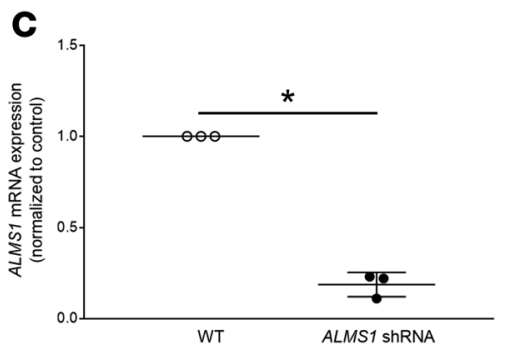
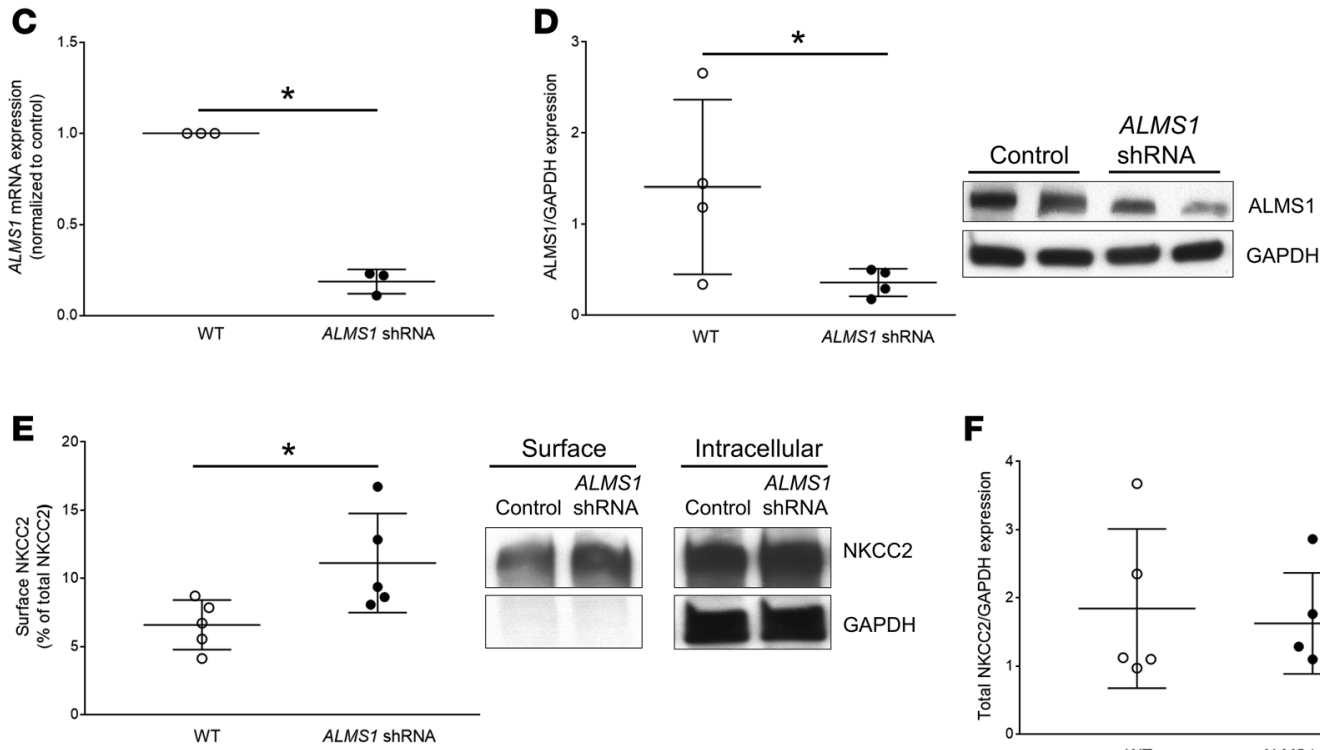

$\mathbf{F}$
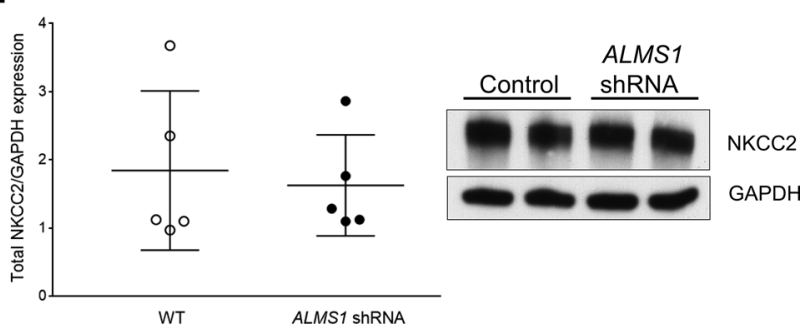

Figure 7. Surface NKCC2 expression is higher in TALs from ALMS1-deleted rats. (A) NKCC2 surface fraction in thick ascending limbs (TALS) from ALMS1$\mathrm{KO}$ rats was higher (ALMS1-KO, 13.8\% $\pm 1.2 \%$, vs. WT, $8.1 \% \pm 1.1 \%, n=6 ;{ }^{*} P<0.05$ ). The lanes were run on the same gel but were noncontiguous. (B) Total NKCC2 expression normalized by the housekeeping control protein GAPDH in TALs from WT and ALMS1-KO rats was similar (normalized value ALMS1-KO, $0.88 \pm 0.07$, vs. WT, $1, n=5$ ). (C) ALMS1 mRNA expression in TALs from normal Sprague Dawley rats measured 7 days after in vivo transduction of $A L M S 1$ shRNA in rat outer medulla was decreased compared with control (normalized value ALMS1 shRNA, $0.19 \pm 0.04$, vs. control, $1, n=3 ;{ }^{*} P<0.01$ ). (D) ALMS1 protein expression normalized to GAPDH (different exposure) in TALs from normal Sprague Dawley rats measured 7 days after in vivo transduction of $A L M S 1$ shRNA was decreased, indicating effective knock down of $A L M S 1$ in rat outer medulla (normalized value $A L M S 1$ shRNA, $0.39 \pm 0.2$, vs. control, $1, n=4$; ${ }^{*} P<$ 0.05). (E) Surface NKCC2 expression measured by surface biotinylation in TALs from normal Sprague Dawley rat kidney transduced with ALMS1 shRNA was enhanced compared with kidney injected with control (ALMS1 shRNA, $11.1 \% \pm 1.6 \%$, vs. control, $6.6 \% \pm 0.8 \%, n=5$; ${ }^{*} P<0.05$ ). (F) Total NKCC2 expression normalized to GAPDH in TALs from normal Sprague-Dawley rat kidney transduced with ALMS1 shRNA was similar to control injected kidney (normalized value ALMS1 shRNA, $0.93 \pm 0.07$, vs. control, $1 ; n=5$ ). Values represent mean $\pm \mathrm{SEM}$, and statistical analysis was performed with 2 -tailed Student's $t$ test.

specific protein-to-protein interactions. $M A L / V I P 17$-overexpressing transgenic mice presented only with an increase in NKCC2 phosphorylation and NKCC2 glycosylation but no effect was reported for renal ion transport in these mice. In the present study, we found that deletion of ALMS1 in rats causes higher NKCC2 surface expression, accompanied with high NKCC2-mediated $\mathrm{NaCl}$ transport and higher BP. Since NKCC2 is known to be stimulated by phosphorylation, we measured this and found no differences in NKCC2 phosphorylation at either pThr (aa 96,101) or pSer (aa 126) between the groups (Supplemental Figure 4). Thus, our data show that ALMS1 is primarily involved in NKCC2 endocytosis and not in the regulation of NKCC2 phosphorylation.

The exact mechanism by which ALMS1 mediates NKCC2 endocytosis remains unclear. NKCC2 is known to undergo endocytosis by 2 mechanisms, a clathrin-mediated and a lipid raft-dependent pathway (45), to maintain NKCC2 surface levels. In this study, we show that gene deletion of ALMS1 decreases NKCC2 endocytosis. To gain insight into the role of ALMS1, we identified proteins that may be part of the NKCC2 endocytic protein network. The carboxyl terminus of ALMS1 pulled down flotillin-2 (FLOT2) (Figure 1E), a lipid raft marker protein and Annexin A2 (ANXA2) (Figure 1E), which was shown to be important for lipid raft-associated apical trafficking of NKCC2 (46). This suggested 

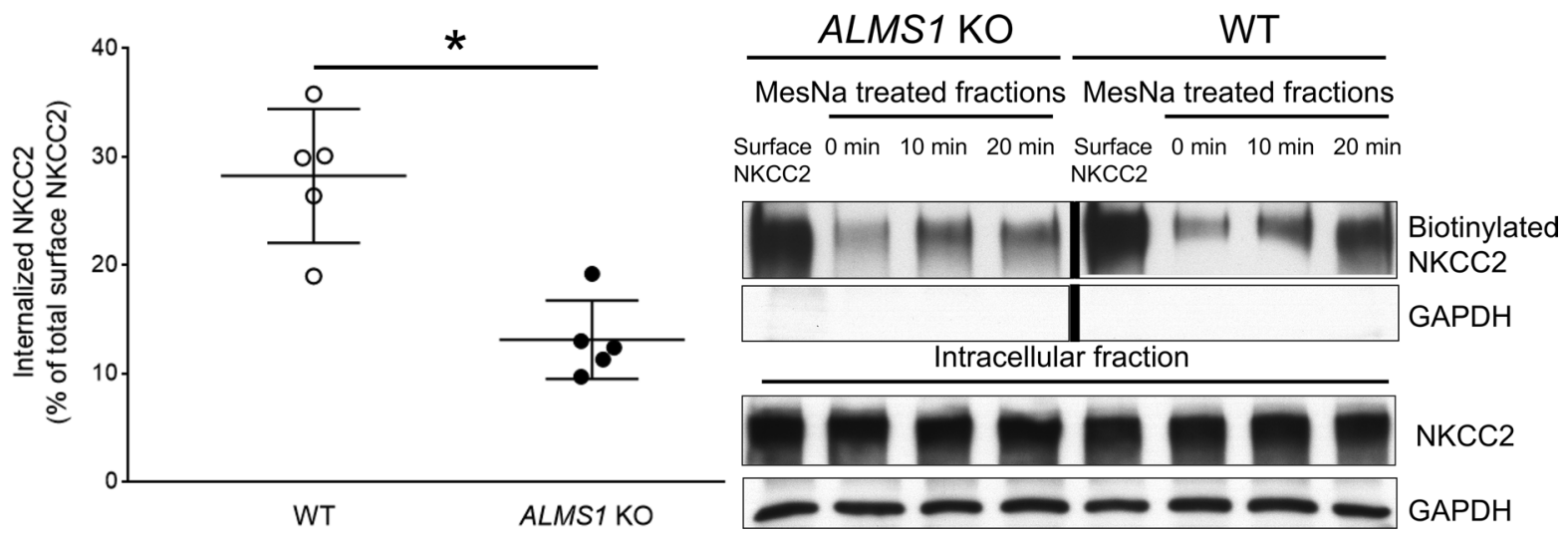

Figure 8. NKCC2 endocytosis is decreased in TALs from ALMS1-KO rats. Representative Western blot shows a lower internalized NKCC2 fraction after warming the thick ascending limbs (TALs) from ALMS1-KO at $37^{\circ} \mathrm{C}$ for 20 minutes. NKCC2 endocytosis measured as a percent of total surface NKCC2 was lower in TALs from ALMS1-KO rats (ALMS1-KO, 13.1\% $\pm 1.4 \%$, vs. WT, 28.2\% $\pm 2.8 \%$ after 20 minutes, $n=5 ;{ }^{*} P<0.05$ ). The WT and ALMS1-KO surface NKCC2 lanes were run on the same gel but were noncontiguous. Values represent mean \pm SEM, and statistical analysis was performed with 2-tailed Student's $t$ test.

that ALMS1 may be involved in lipid raft-dependent NKCC2 endocytosis. However, we also pulled down phosphatidylinositol binding clathrin assembly protein (PICALM) (Figure 1E), which mediates clathrin-dependent endocytosis and targeting of clathrin-coated vesicles to early and late endosomes (47). Together, our data suggest that ALMS1 may be a common scaffold that recruits proteins involved in determining lipid raft- and/or clathrin-dependent endocytosis. While this question is unresolved, the network analysis of C-ALMS1 interacting proteins further supports a role for ALMS1 in mediating membrane protein endocytosis.

Overall, our data provides the first evidence to our knowledge that ALMS1 is involved in the regulation of renal sodium transport and, therefore, $\mathrm{BP}$ regulation such that ALMS1-KO rats are hypertensive and have decreased ability to excrete a volume load. The mechanism for this defect involves decreased NKCC2 endocytosis, which causes accumulation of NKCC2 at the apical surface and higher TAL NaCl transport. It is important to note that other mechanisms may also contribute toward higher BP observed in the ALMS1-KO rats. ALMS1 was not exclusively located in the TAL, suggesting that it may regulate trafficing of other renal sodium transporters. Although CKD and hypertension are common in Alström syndrome patients, the causal nexus among these conditions is unknown. It is possible that hypertension accompanies the development of fibrosis and CKD due to the loss-of-functional nephrons over time in these patients. Nonetheless, the existing GWAS data linking SNPs in ALMS1 with decreased renal function, and the severe phenotype observed in Alström syndrome patients $(35,36)$, along with our data suggest that $A L M S 1$ is an important gene for renal function and BP control and is of high relevance in the general population.

\section{Methods}

Animals. In vivo transduction of ALMS1 shRNA in the outer medulla was performed in 90-110 g male Sprague Dawley rats (Charles River Laboratories) as reported previously (33). The homozygous ALMS1KO rats used were males of 6-12 weeks of age. Age-matched littermate controls were male Dahl salt-sensitive rats. Animals were fed a standard $\operatorname{diet}\left(0.22 \% \mathrm{Na}^{+}, 1 \% \mathrm{~K}^{+}\right)$from Envigo.

Generation of ALMS1-KO rats. Frame-shift deletion of $17 \mathrm{bp}$ in exon 1 (containing Nci1 restriction site) of the ALMS1 gene was facilitated by injecting zinc finger nucleases targeting the sequence $5^{\prime}$ - CCCGCCTCCGACTCCGCCtccgtcCTCCCGGCACCAGTA -3 ' into Dahl SS/JrHsdMcwi rat embryos. This deletion caused a frame shift leading to a premature stop codon in exon 2. Confirmation of deletion was done by PCR using exon 1 specific primers: Forward primer, 5' - AGAGGAAGAGTTGGAAGGGG - 3'; reverse primer, $5^{\prime}$ - ATACATAGGCAGAGCGACCC - 3', followed by Nci1 restriction digestion. This was performed in collaboration with the GERRC at the Medical College of Wisconsin.

Antibody and reagents. The anti-NKCC2 antibody was generated in rabbit against a carboxy-terminal sequence as described before $(19,32)$. pThr (aa 96, 101) NKCC2, pSer (aa 126), and extracellular NKCC2 antibodies were used as previously described $(15,33,48)$. Antibodies against carboxyl-terminus ALMS1 


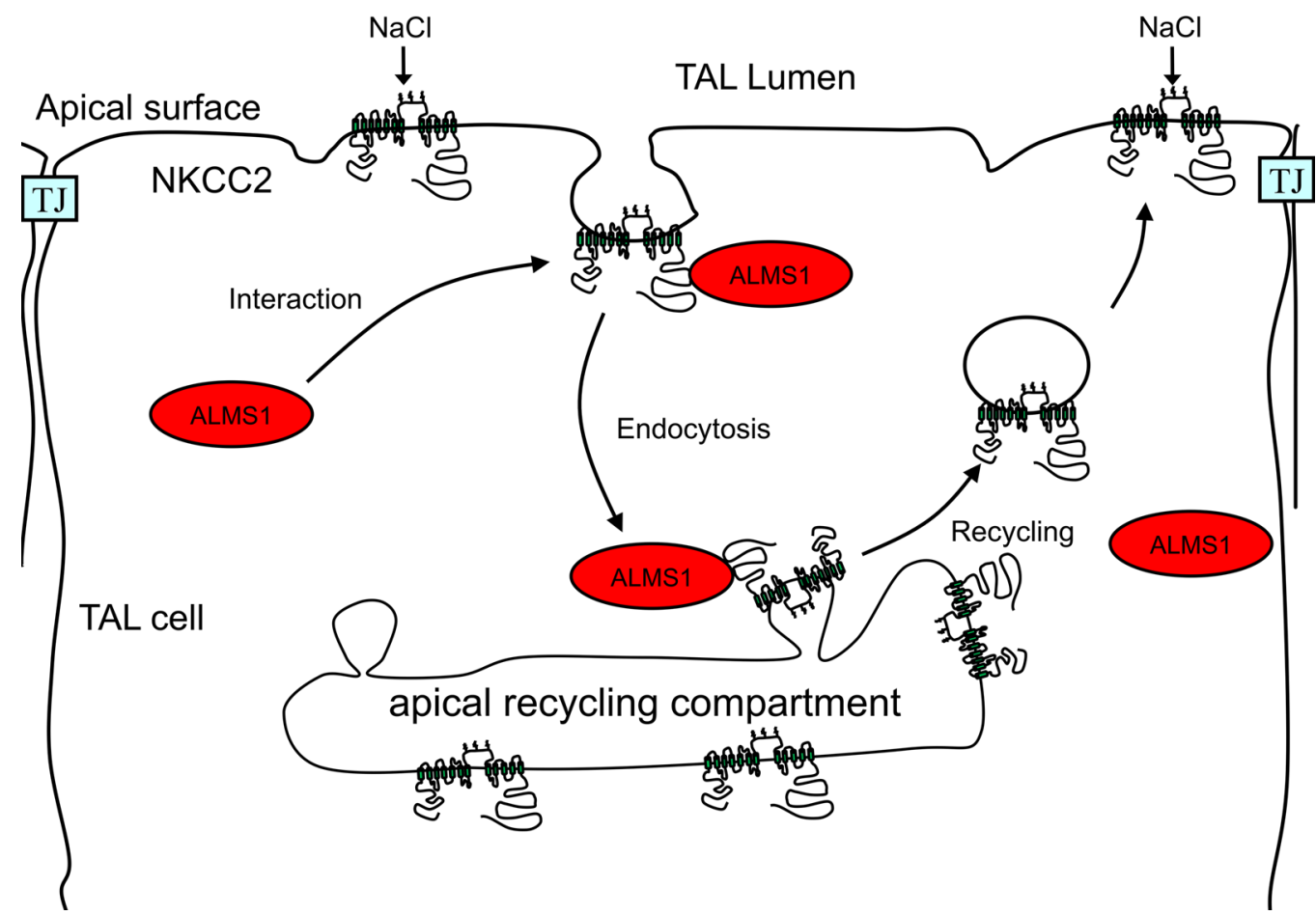

Figure 9. Working model. Diagrammatic representation of the role of ALMS1 in endocytosis of NKCC2. At some point of the endocytic process, the carboxyl terminus of ALMS1 (C-ALMS1) interacts with the C2-terminus of NKCC2 (C2-NKCC2) to accelerate its endocytosis and regulate NKCC2 levels at the apical surface.

was generated in rabbit and obtained by J.K. Naggert (5). Another affinity-purified ALMS1 antibody was generated against a short peptide epitope in the C-terminus of ALMS1: CEARLEEDSDVTSSSEEKAKE (similar to antibody previously published; ref. 5). Another ALMS1 antibody was generated against amino acids (aa 2,174-2,296) of mouse ALMS1 (Genscript Inc.) (Supplemental Figure 1). GAPDH antibody was purchased from MilliporeSigma (AB2302). ZO-1 antibody was purchased from Thermo Fisher Scientific (ZO1-1A12). Reagents for surface biotinylation were purchased from Thermo Fisher Scientific and, for GST-pull down, were from GE Life Sciences. Bumetanide was purchased from MilliporeSigma.

Transduction of cells. NRK 52E cells (American Type Culture Collection; ATCC) were grown in a 60-mm cell culture dish with DMEM (Invitrogen) with 5\% FBS (Invitrogen). Once confluent, the cells were transduced with eGFP or eGFP-NKCC2 adenovirus (Viraquest) as described previously (48) at $1 \times 10^{6}$ plaque forming units $(\mathrm{PFU} / \mathrm{ml})$ in serum free DMEM. After 2 hours, $5 \%$ serum was added to inactivate viruses, and cells were incubated for 48 hours at $37^{\circ} \mathrm{C}$.

Suspensions of medullary TALs. TAL suspensions were obtained as described previously. Animals were anesthetized with ketamine (VetOne, 501072) and xylazine (Santa Cruz Biotechnology Inc., sc362950Rx) mixture i.p. and kidneys perfused via the descending aorta with $0.001 \%$ collagenase. Kidneys were harvested, and the outer medulla was dissected and digested in $0.1 \%$ collagenase at $37^{\circ} \mathrm{C}$ for 30 minutes with oxygenation every 5 minutes, followed by mechanical stirring on ice for 30 minutes. The suspension was then filtered through a $250-\mu$ m nylon mesh.

Steady-state surface biotinylation assay. Steady-state surface NKCC2 was measured in suspensions of TALs as described previously (19). TALs were biotinylated at $4^{\circ} \mathrm{C}$ with NHS-SS-biotin $(0.9 \mathrm{mg} / \mathrm{ml})$ and then washed and lysed. Extracted proteins were incubated with steptavidin-agarose beads (Pierce Biotechnology) at $4^{\circ} \mathrm{C}$ overnight to separate the biotinylated protein fraction (surface protein fraction) from the nonbiotinylated protein fraction (intracellular protein fraction). Beads were washed and proteins were extracted from beads by warming at $37^{\circ} \mathrm{C}$. Proteins were resolved by SDS-PAGE ( $\% \%$ gels). NKCC2 and GAPDH were detected by Western blot. Surface NKCC2 was calculated ( $30 \mu \mathrm{g}$ fraction) as a percentage of total NKCC2 (sum of surface and intracellular NKCC2 fraction).

In vivo gene silencing. The target sequence for ALMS1 silencing was a 29-nucleotide sequence from the rat ALMS1 gene: $5^{\prime}$ - TCCTGAATCAGGTGACCAGAAGACTCATT - 3'. The sense and antisense 
sequences are spaced by a loop sequence (TCAAGAG). This shRNA sequence was tested by transfection in NRK-52E cells from the ATCC (data not shown). mRNA expression of ALMS1 and GAPDH was monitored by real-time PCR. After the efficacy of the shRNA was tested, the shRNA was subcloned between the 5' AscI and 3' HindIII sites in the Adenovector-pMIGHTY (Viraquest) for production of adenoviral particles, as recently described (33). The adenoviral ALMS1 shRNA was injected with 3-mm calibrated needles into the outer medulla of the left rat kidney while the right kidney was injected with the control buffer, as we described before for other genes (49). The kidneys were harvested 7 days later to obtain TAL suspensions, which were lysed and resolved by SDS-PAGE in 4\% gels or $6 \%$ gels for the detection of ALMS1 and NKCC 2 by Western blot, respectively. Surface NKCC2 was calculated as a percentage of total NKCC2 (sum of surface and intracellular NKCC2 fraction).

Endocytosis assay of NKCC2 in TALs. NKCC2 endocytosis was measured as described previously (19). TALs were biotinylated at $4^{\circ} \mathrm{C}$. Suspensions were split into 4 , and the last 3 fractions were warmed to $37^{\circ} \mathrm{C}$ for 0,10 , and 20 minutes to allow endocytosis of surface proteins. The TALs were rapidly cooled to halt protein trafficking. TALs were then treated for 30 minutes at $4^{\circ} \mathrm{C}$ with the membrane-impermeable reducing agent $\mathrm{MesNa}(50 \mathrm{mM})$ to strip biotin from surface proteins while protecting biotinylated proteins that internalized. TALs from all 4 fractions were then lysed, and equal amounts of proteins were incubated with agarose-streptavidin to separate biotinylated proteins that internalized while the first fraction served as baseline surface NKCC2. Proteins were then resolved by SDS-PAGE, and NKCC2 was detected by Western blot. Internalized NKCC2 was measured as a percentage of total (100\%) biotinylated surface NKCC2 fraction.

GST pull down. A GST fusion protein consisting of GST fused to the 71-amino acid domain in NKCC2 that is shown to be important for apical targeting of NKCC2 (GST-C2-NKCC2) was obtained from J.K. Naggert. It was engineered by subcloning the corresponding nucleotides in the sequence of rat NKCC2 downstream of GST in the pGEX-6P-1 vector (GE Healthcare). The GST-alone construct served as the negative control. The constructs were separately transformed by heat shock into competent $E$. coli and induced to express the construct by incubation with $0.18 \mathrm{mM}$ IPTG at $28^{\circ} \mathrm{C}$ for 10 hours. Bacterial proteins were extracted and incubated with glutathione-conjugated sepharose beads (GE Healthcare) to purify the induced protein. Eat TAL protein lysate $(100 \mu \mathrm{g})$ was incubated with purified GST-C2-NKCC2 and GST alone overnight at $4^{\circ} \mathrm{C}$ after preclearing the lysate with GST alone for 1 hour. The beads were washed the next day and submitted to the Proteomics Core of Michigan State University (East Lansing, Michigan, USA) for LC-MS to identify C2-NKCC2 interacting proteins. To determine if C-ALMS1 binds to NKCC2, 2 truncated carboxyl-terminus ALMS1 constructs were used. These truncated constructs correspond to amino acids 2,683-2,932 (referred to as C-ALMS1-B) and 2,988 to 3,251 (referred as C-ALMS1-A) in mouse ALMS1. These constructs were cloned downstream of GST in pGEX-6P-1 vector, while GST-alone served as a negative control. Purification of the induced proteins and GST pull-down assay was performed as mentioned above. The pull-down proteins were eluted from glutathione-conjugated sepharose beads with $6 \times$ loading buffer containing DTT and $\beta$-mercaptoethanol and resolved by SDS-PAGE. NKCC2 was detected by Western blot.

Immunoprecipitation. NRK-52E cells were transduced with adenoviruses coding for eGFP alone or NKCC2-eGFP for 48 hours. Cells were then lysed and protein lysates incubated with $50 \mu 1$ magnetic control or magnetic anti-GFP mAb beads from MBL (D15311, Sakae). Captured immunocomplexes were immunoblotted with anti-ALMS1 or -NKCC2 antibodies (in-house).

Ingenuity pathway analysis. TAL proteins pulled down either with GST-C-ALMS1 A/B were identified by LC-MS. These proteins were found to be involved with key biological processes such as cellular assembly and organization, molecular transport, protein trafficking, and clathrin- and caveolar-mediated endocytosis signaling, with an enrichment probability value less than 0.05 , considered statistically significant by Ingenuity Pathway Analysis (IPA, Qiagen Inc.). These proteins were further curated with IPA knowledgebase by IPA analysis for direct and indirect protein-to-protein interactions among themselves.

Histology and IHC. Kidneys were fixed by perfusing the rats with $4 \%$ paraformaldehyde through the abdominal aorta. The kidneys were then stored in 10\% formalin for a day before embedding and processing the tissue by the Biobank and Correlative Science Core at Wayne State University. Sections of the kidney (5- $\mu \mathrm{m}$ thick) were processed for H\&E staining and immunostaining. For colabeling of NKCC2 and ALMS1 in kidney sections, paraffin-embedded slices were first deparaffinized with xylene and then hydrated gradually through $100 \%$ ethanol to distilled water. Heat-induced epitope retrieval was used to unmask the antigens followed by incubation with $1 \%$ BSA to block nonspecific binding. Slides were incubated with 
1:50 dilution of NKCC2 primary antibody for 1 hour at room temperature (RT). This was followed by 1:100 of Alexa-Fluor 488 goat anti-rabbit IgG (Thermo Fisher Scientific, A11034) for 1 hour at RT. To facilitate labeling of ALMS1, biotinylated ALMS1 antibody was prepared using the antibody biotinylation kit (Pierce, Thermo Fisher Scientific). The slides were incubated with 1:50 dilution of streptavidin prior to incubating them with ALMS1 biotinylated antibody at 1:20 dilution at RT for 1 hour to block nonspecific binding. This was followed by incubating with 1:100 of streptavidin Alexa-Fluor 647 for 1 hour at RT. Finally, the slides were counter-stained with DAPI at 1:2,000 dilution for 5 minute at RT. For staining cilia in kidney sections, slides were incubated with 1:250 dilution of mouse anti-acetylated $\alpha$-tubulin antibody (Abcam, ab24610) followed by 1:200 dilution of Alexa-Fluor 488 goat anti-mouse IgG (Thermo Fisher Scientific) for 1 hour at RT. Slides were mounted and imaged by Leica multiphoton microscope TCS SP8 MP (Leica Microsystems) equipped with a tunable white light laser and 6 chromatic detector channels.

TAL primary culture. Primary culture of rat TAL was done as previously described (33) following institutional and national guidelines for care and use of laboratory animals. Sprague-Dawley rats weighing 100$120 \mathrm{~g}$ were used. Kidneys were perfused with $0.1 \%$ collagenase (MilliporeSigma), and the outer medulla was dissected. Individual TAL cells were obtained from TAL suspensions after digestion of the tubules with $0.1 \%$ collagenase, $0.25 \%$ trypsin (MilliporeSigma), and $0.0021 \%$ DNase (MilliporeSigma). Single TAL cells were isolated by density gradient centrifugation in 35\% Percoll (MilliporeSigma), washed, and seeded in permeable supports (Corning) coated with basement membrane extract (Trevigen). Cells were grown for 4 days in DMEM (low glucose, no phenol red, no glutamine) supplemented with $1 \% \mathrm{FBS}$, a penicillin-streptomycin mix, and insulin-transferrin-selenium (Invitrogen) at $37^{\circ} \mathrm{C}$ and $5 \% \mathrm{CO}_{2}$ until $90 \%$ confluent.

Coimmunostaining in TAL primary culture. TAL primary cells that achieved $90 \%$ confluence on permeable supports were cooled to $4^{\circ} \mathrm{C}$. Live cells were incubated with $5 \%$ BSA (Equitech-Bio) in PBS (Invitrogen) for 20 minutes at $4^{\circ} \mathrm{C}$. Surface NKCC2 was labeled with an antibody (1:100) directed toward extracellular epitope of NKCC2 added to the apical compartment of the transwells containing live cells for 2 hours at $4^{\circ} \mathrm{C}$ (33). The cells were then washed and incubated with an Alexa-Fluor 488conjugated anti-rabbit antibody for 1 hour at $4^{\circ} \mathrm{C}$ in the apical compartment of the transwell. The cells were washed and warmed to $37^{\circ} \mathrm{C}$ for allowing internalization of the surface labeled NKCC2. The cells were then fixed with $4 \%$ paraformaldehyde for 30 minutes at $4^{\circ} \mathrm{C}$. Fixed cells were washed and incubated with 5\% BSA in PBS for 20 minutes at RT. To facilitate labeling of ALMS1, biotinylated ALMS1 antibody was prepared using the antibody biotinylation kit (Pierce, Thermo Fisher Scientific) and was then subsequently added to the apical compartment for 1 hour at RT. The cells were washed and then incubated with streptavidin Alexa-Fluor 647 for 1 hour at RT. The cells were then washed and a mouse primary antibody against zonula occludens-1 (ZO-1) was added to the cells, which were incubated for 1 hour at RT followed by the addition of an Alexa-Fluor 568-conjugated secondary antimouse antibody (Thermo Fisher Scientific, A11031) for 1 hour. The membranes were then washed, cut, and mounted on a glass slide. Glass slides were imaged by Leica multiphoton microscope TCS SP8 MP (Leica Microsystems). For Z-section confocal imaging, we used this confocal system at $\times 60$, 1.4 NA. Z-sections were obtained at $0.25 \mu \mathrm{m}$ steps for $8.75 \mu \mathrm{m}$ total Z-distance ( 35 slices). The colocalization pixels were identified by using a Mander's overlap coefficient $\geq 0.9$.

Labeling of ALMS1 in isolated perfused rat TAL. Isolated perfused rat TALs were fixed with 4\% paraformaldehyde and then blocked with 2.5\% BSA. We then labeled TALs with anti-ALMS1 antibody in 2.5\% BSA followed by Alexa-Fluor 488 anti-rabbit IgG highly adsorbed at 1:100 in 2.5\% BSA. Images were acquired using a laser scanning confocal microscopy system (Visitech). Images were acquired at 100× (1.3 NA).

$B P$ measurements. Systolic arterial pressure was measured by implanting radio telemetry device in femoral artery of rats anesthetized with a mixture of xylazine and ketamine i.p. Rats were allowed to recover from the surgery for a week before recording the actual BP and heart rate measurements by radiotelemetry. The measurements reported are average readings from 3 consecutive days. In the salt-sensitivity protocol, systolic arterial pressure was measured by tail cuff plethysmography in awake rats for testing salt-induced changes in BP. Rats were trained 3 times a week for 2 weeks prior to baseline measurements on normal salt diet. $\mathrm{BP}$ was measured 4 and 7 days after switching rats to high-salt intake $(0.5 \% \mathrm{NaCl}$ in drinking water) to determine salt sensitivity.

Urine and ion excretion measurements. Rats were placed in metabolic cages with free access to food and water. The rats were allowed to get adjusted to the new cage for 3 days, after which urine samples were collected. Urine volume was recorded and urine osmolality was measured by freezing point depression with Advanced 
Model 3300 Micro Osmometer (Advanced Instruments Inc.). Plasma electrolytes and urine $\mathrm{Na}^{+}$was measured in Nova 1+ Analyzer (Nova Biomedical). For bumetanide response experiments, a different group of rats were trained to eat all of their food between 6 p.m. and 9 a.m. the next day. At 10 a.m., rats were given $1 \mathrm{~g}$ chocolate pudding and were trained to eat chocolate pudding within 10 minutes for 5 days. On day 5 , urine was collected until 8 hours after the rats were given chocolate pudding to record baseline urine volume and $\mathrm{Na}^{+}$excretion. On day $6,5 \mathrm{mg} / \mathrm{kg}$ bumetanide (as per the average weight of the WT rats) was mixed with the chocolate pudding, and urine was collected to measure urine volume and urinary sodium content until 8 hours after the rats were treated with bumetanide.

GFR and renal blood flow measurement. Rats were fasted overnight, and on the following day, they were anesthetized and their femoral vein was cannulated for bolus administration ( $3 \mu \mathrm{l} / \mathrm{g}$ body weight) of $1 \mathrm{mg} /$ $\mathrm{ml} \mathrm{FITC-inulin} \mathrm{followed} \mathrm{by} \mathrm{its} \mathrm{constant} \mathrm{infusion} \mathrm{at} \mathrm{a} \mathrm{rate} \mathrm{of} 0.15 \mu \mathrm{l} / \mathrm{min} / \mathrm{g}$ body weight for measurement of GFR by measuring clearance of FITC-inulin. A midline incision was made to expose the left kidney and the bladder. A flow probe was placed on the renal artery to measure renal blood flow. The bladder was catheterized to collect urine. Plasma and urine FITC-inulin fluorescence was assessed with a Synergy H1 Microplate Reader (BioTek) to measure plasma and urine inulin concentrations, as described previously (50).

Plasma insulin measurement. Whole blood was collected from the jugular vein of rats and was immediately mixed with appropriate volume of EDTA (5:1 ratio) to prevent blood coagulation. Blood was then spun at $200 \mathrm{~g}$ for 20 minutes to collect plasma. Plasma samples were used to measure insulin concentration using Enzyme immunoassay kit from Cayman.

Volume/saline expansion (VE). Rats were anesthetized with inactin i.p., and a femoral artery and vein were cannulated with PE-50 tubing. Femoral arterial pressure was measured with a pressure transducer. The bladder was exposed through a suprapubic incision and was cannulated with a needle attached to PE-50 tubing. Isotonic saline was infused i.v. to administer $3 \mathrm{ml} / 100 \mathrm{~g}$ body weight in 30 minutes (3\% $\mathrm{VE})$, after which VE was maintained by infusing saline at a rate averaging $0.15 \mu \mathrm{l} / \mathrm{min} / \mathrm{g}$ body weight. Following a 30-minute recovery period after surgery, urine was collected at 2 consecutive 30-minute intervals (time points 30 minutes and 60 minutes) for baseline measurements of urine volume and urinary sodium excretion and then at 5 consecutive 30-minute intervals (time points 90-210 minutes) following $3 \% \mathrm{VE}$. The data points plotted represent values for total urine volume and urinary sodium excretion measured over 30 minutes starting from the preceding data point. These physiological parameters measured after 3\% VE were first normalized to their respective baseline values at 60 minutes. Then, their cumulative values between time points 60 minutes and 210 minutes (represented by light and dark gray shaded regions) (Figure 6, A and B) were calculated and plotted in the corresponding bar graph.

Statistics. Results are expressed as mean \pm SEM. Single intergroup comparisons between 2 groups were performed with a 2-tailed Student's $t$ test. Two-way ANOVA was used to determine differences between means in 2 treatments and groups. Post hoc analysis was performed when differences between means was found using the Bonferroni correction for multiple comparisons. $P<0.05$ was considered statistically significant.

Study approval. All procedures involving live animals were approved by the IACUC of the Henry Ford Hospital and conducted following its guidelines.

\section{Author contributions}

ABJ designed research studies, conducted experiments, acquired data, analyzed data, wrote the manuscript, and revised the manuscript. PSC conducted 1 experiment/protocol, analyzed the corresponding data, and edited the manuscript. KKM conducted 1 experiment/protocol and acquired and analyzed the corresponding data. TDL conducted 1 experiment/protocol. ID analyzed data by IPA. DM performed 1 experiment. JKN reviewed/edited manuscript, provided reagents, and provided antibodies against carboxyl-terminus ALMS1. WHB designed research studies and reviewed/edited manuscript. PAO designed research studies and edited and approved the final version of the manuscript.

\section{Acknowledgments}

This work was supported in part by NIH grant 1R01DK107263 01A1, American Heart Association Grantin-Aid to PAO, and American Heart Association - Predoctoral fellowship award 16PRE27510032 to ABJ. We would like to acknowledge Gustavo Ares and D'Anna Potter for their technical assistance. 
Address correspondence to: Pablo A. Ortiz, 2799 West Grand Boulevard, E\&R building, Room 7099, Detroit, Michigan 48202, USA. Phone: 313.916.7164; Email: portiz1@hfhs.org.

PSC's present address is: Department of Ophthalmology, Margaret Dyson Vision Research Institute, Weill Cornell Medical College, New York, New York, USA.

ABJ's present address is: Department of Pharmacology, UT Southwestern Medical Center, Dallas, Texas, USA.

1. Köttgen A et al. Multiple new loci associated with kidney function and Chronic Kidney Disease: The CKDGen consortium Nat Genet. 2010;42(5):376-384

2. Chambers JC, et al. Genetic loci influencing kidney function and chronic kidney disease. Nat Genet. 2010;42(5):373-375

3. Böger CA, et al. Association of eGFR-Related Loci Identified by GWAS with Incident CKD and ESRD. PLoS Genet. 2011;7(9):e1002292.

4. Barkley RA, et al. Positional identification of hypertension susceptibility genes on chromosome 2. Hypertension. 2004;43(2):477-482.

5. Collin GB, et al. The Alström syndrome protein, ALMS1, interacts with $\alpha$-actinin and components of the endosome recycling pathway. PLoS One. 2012;7(5):e37925.

6. Collin GB, et al. Alms1-disrupted mice recapitulate human Alström syndrome. Hum Mol Genet. 2005;14(16):2323-2333.

7. Leitch CC, Lodh S, Prieto-Echagüe V, Badano JL, Zaghloul NA. Basal body proteins regulate Notch signaling through endosomal trafficking. J Cell Sci. 2014;127(pt 11):2407-2419.

8. Favaretto F, et al. GLUT4 defects in adipose tissue are early signs of metabolic alterations in Alms1GT/GT, a mouse model for obesity and insulin resistance. PLoS One. 2014;9(10):e109540.

9. Schulz TW, et al. Actin/ $\alpha$-actinin-dependent transport of AMPA receptors in dendritic spines: role of the PDZ-LIM protein RIL. J Neurosci. 2004;24(39):8584-8594.

10. Piirainen H, Taura J, Kursula P, Ciruela F, Jaakola VP. Calcium modulates calmodulin/ $\alpha$-actinin 1 interaction with and agonist-dependent internalization of the adenosine A2A receptor. Biochim Biophys Acta Mol Cell Res. 2017;1864(4):674-686.

11. Arasaki K, et al. A new role for RINT-1 in SNARE complex assembly at the trans-Golgi network in coordination with the COG complex. Mol Biol Cell. 2013;24(18):2907-2917.

12. Provance DW, Addison EJ, Wood PR, Chen DZ, Silan CM, Mercer JA. Myosin-Vb functions as a dynamic tether for peripheral endocytic compartments during transferrin trafficking. BMC Cell Biol. 2008;9:44.

13. Aviv A, Hollenberg NK, Weder A. Urinary potassium excretion and sodium sensitivity in blacks. Hypertension. 2004;43(4):707-713.

14. Jung J, Basile DP, Pratt JH. Sodium reabsorption in the thick ascending limb in relation to blood pressure: a clinical perspective. Hypertension. 2011;57(5):873-879.

15. Haque MZ, Ares GR, Caceres PS, Ortiz PA. High salt differentially regulates surface NKCC2 expression in thick ascending limbs of Dahl salt-sensitive and salt-resistant rats. Am J Physiol Renal Physiol. 2011;300(5):F1096-F1104.

16. Meade $\mathrm{P}$, et al. cAMP-dependent activation of the renal-specific $\mathrm{Na}^{+}-\mathrm{K}^{+}{ }^{2} \mathrm{Cl}^{-}$cotransporter is mediated by regulation of cotransporter trafficking. Am J Physiol Renal Physiol. 2003;284(6):F1145-F1154.

17. Carmosino M, et al. MAL/VIP17, a new player in the regulation of NKCC2 in the kidney. Mol Biol Cell. 2010;21(22):3985-3997.

18. Carmosino M, Giménez I, Caplan M, Forbush B. Exon loss accounts for differential sorting of Na-K-Cl cotransporters in polarized epithelial cells. Mol Biol Cell. 2008;19(10):4341-4351.

19. Ares GR, Ortiz PA. Constitutive endocytosis and recycling of NKCC2 in rat thick ascending limbs. Am J Physiol Renal Physiol. 2010;299(5):F1193-F1202.

20. Akhtar N, Hotchin NA. RAC1 regulates adherens junctions through endocytosis of E-cadherin. Mol Biol Cell. 2001;12(4):847-862.

21. Cremona ML, et al. Flotillin-1 is essential for PKC-triggered endocytosis and membrane microdomain localization of DAT. Nat Neurosci. 2011;14(4):469-477.

22. Schneider A, et al. Flotillin-dependent clustering of the amyloid precursor protein regulates its endocytosis and amyloidogenic processing in neurons. J Neurosci. 2008;28(11):2874-2882.

23. de Graauw M, et al. Annexin A2 depletion delays EGFR endocytic trafficking via cofilin activation and enhances EGFR signaling and metastasis formation. Oncogene. 2014;33(20):2610-2619.

24. Rescher U, Zobiack N, Gerke V. Intact $\mathrm{Ca}(2+)$-binding sites are required for targeting of annexin 1 to endosomal membranes in living HeLa cells. J Cell Sci. 2000;113(pt 22):3931-3938.

25. Thomas RS, Henson A, Gerrish A, Jones L, Williams J, Kidd EJ. Decreasing the expression of PICALM reduces endocytosis and the activity of $\beta$-secretase: implications for Alzheimer's disease. BMC Neurosci. 2016;17(1):50.

26. Hussain KM, Leong KL, Ng MM, Chu JJ. The essential role of clathrin-mediated endocytosis in the infectious entry of human enterovirus 71. J Biol Chem. 2011;286(1):309-321.

27. Han K, et al. Regulated RalBP1 binding to RalA and PSD-95 controls AMPA receptor endocytosis and LTD. PLoS Biol. 2009;7(9):e1000187.

28. Kumari S, Mayor S. ARF1 is directly involved in dynamin-independent endocytosis. Nat Cell Biol. 2008;10(1):30-41.

29. Akhtar N, Streuli CH. An integrin-ILK-microtubule network orients cell polarity and lumen formation in glandular epithelium. Nat Cell Biol. 2013;15(1):17-27.

30. Wang YJ, et al. Prohibitin is involved in the activated internalization and degradation of protease-activated receptor 1. Biochim Biophys Acta. 2014;1843(7):1393-1401.

31. Kim JH, Lee-Kwon W, Park JB, Ryu SH, Yun CH, Donowitz M. Ca(2+)-dependent inhibition of $\mathrm{Na}^{+} / \mathrm{H}^{+}$exchanger $3(\mathrm{NHE} 3)$ requires an NHE3-E3KARP- $\alpha$-actinin-4 complex for oligomerization and endocytosis. J Biol Chem. 2002;277(26):23714-23724. 
32. Ortiz PA. cAMP increases surface expression of NKCC2 in rat thick ascending limbs: role of VAMP. Am J Physiol Renal Physiol. 2006;290(3):F608-F616

33. Caceres PS, Mendez M, Ortiz PA. Vesicle-associated membrane protein 2 (VAMP2) but Not VAMP3 mediates cAMP-stimulated trafficking of the renal $\mathrm{Na}^{+}-\mathrm{K}^{+}{ }^{2} \mathrm{Cl}^{-}$co-transporter NKCC2 in thick ascending limbs. J Biol Chem. 2014;289(34):23951-23962.

34. Takahashi N, Chernavvsky DR, Gomez AR, Igarashi P, Gitelman HJ Smithies O. Uncompensated polyuria in a mouse model of Bartter's syndrome. Proc Natl Acad Sci U S A. 2000;97(10):5434-5439.

35. Marshall JD, et al. New Alström Syndrome phenotypes based on the evaluation of 182 cases. Arch Intern Med. 2005;165(6):675-683.

36. Marshall JD, Maffei P, Collin GB, Naggert JK. Alström Syndrome: genetics and clinical overview. Curr Genomics. 2011;12(3):225-235.

37. Padmanabhan $\mathrm{S}$, et al. Genome-wide association study of blood pressure extremes identifies variant near UMOD associated with hypertension. PLoS Genet. 2010;6(10):e1001177.

38. Pattaro C, et al. A meta-analysis of genome-wide data from five European isolates reveals an association of COL22A1, SYT1, and GABRR2 with serum creatinine level. BMC Med Genet. 2010;11:41.

39. Gudbjartsson DF, et al. Association of variants at UMOD with chronic kidney disease and kidney stones-role of age and comorbid diseases. PLoS Genet. 2010;6(7):e1001039.

40. Köttgen A, et al. Multiple loci associated with indices of renal function and chronic kidney disease. Nat Genet. 2009;41(6):712-717.

41. Pattaro C, et al. Genome-wide association and functional follow-up reveals new loci for kidney function. PLoS Genet. 2012;8(3):e1002584.

42. Trudu M, et al. Common noncoding UMOD gene variants induce salt-sensitive hypertension and kidney damage by increasing uromodulin expression. Nat Med. 2013;19(12):1655-1660.

43. Knorz VJ, et al. Centriosomal association of ALMS1 and likely centrosomal functions of the ALMS motif-containing proteins C10orf90 and KIAA1731. Mol Biol Cell. 2010;21(21):3617-3629.

44. Li G, et al. A role for Alström syndrome protein, Alms1, in kidney ciliogenesis and cellular quiescence. PLoS Genet. 2007;3(1):e8.

45. Ares GR, Ortiz PA. Dynamin 2, clathrin, and lipid rafts mediate endocytosis of the apical $\mathrm{Na}^{+} / \mathrm{K}^{+} /{ }^{2} \mathrm{Cl}^{-}$cotransporter $\mathrm{NKCC} 2$ in thick ascending limbs. J Biol Chem. 2012;287(45):37824-37834.

46. Dathe C, et al. Annexin A2 mediates apical trafficking of renal $\mathrm{Na}^{+}-\mathrm{K}^{+}-2 \mathrm{Cl}^{-}$cotransporter. J Biol Chem. 2014;289(14):9983-9997.

47. Rai S, et al. Clathrin assembly protein CALM plays a critical role in KIT signaling by regulating its cellular transport from early to late endosomes in hematopoietic cells. PLoS One. 2014;9(10):e109441.

48. Jaykumar AB, Caceres PS, Sablaban I, Tannous BA, Ortiz PA. Real-time monitoring of NKCC2 endocytosis by total internal reflection fluorescence (TIRF) microscopy. Am J Physiol Renal Physiol. 2016;310(2):F183-F191.

49. Ortiz PA, Hong NJ, Plato CF, Varela M, Garvin JL. An in vivo method for adenovirus-mediated transduction of thick ascending limbs. Kidney Int. 2003;63(3):1141-1149.

50. Sigmon DH, Beierwaltes WH. Influence of nitric oxide derived from neuronal nitric oxide synthase on glomerular filtration Gen Pharmacol. 2000;34(2):95-100. 